Laboratory of Economics and Management Sant'Anna School of Advanced Studies

Via Carducci, 40 - I-56127 PISA (Italy)

Tel. +39-050-883-341 Fax +39-050-883-344

Email: lem@sssup.it Web Page: http://lem.sssup.it

\title{
LEM
}

Working Paper Series

\section{Coordination, Local Interactions and Endogenous Neighborhood Formation}

Giorgio Fagiolo*

${ }^{*}$ St.Anna School of Advanced Studies, Pisa, Italy 


\title{
Coordination, Local Interactions and Endogenous Neighborhood Formation
}

\author{
Giorgio Fagiolo* \\ fagiolo@sssup.it \\ September 2002
}

\begin{abstract}
The paper studies the evolution of coordination in a local interaction model where agents can simultaneously choose the strategy to play in the game and the size of their neighborhood. We focus on pure-coordination games played by agents located on one-dimensional lattices when network externalities become eventually negative as neighborhood sizes increase. We show that the society almost always converge to steady-states characterized by high levels of coordination and small neighborhood sizes. Furthermore, we find that neighborhood adjustment allows for higher coordination than if interaction structures were static and that large populations attain higher coordination provided that average initial neighborhood sizes are not too small. Finally, we explore how long-run patterns of coordination change when: (i) agents possibly enjoy positive network externalities as neighborhood sizes increase; (ii) individuals are placed in higher-dimensional lattices; (iii) agents play coordination games where a trade-off arises between Pareto-efficient and risk-dominant Nash equilibria.
\end{abstract}

Keywords: Learning, Coordination, Nearest-Neighbor Interactions, Endogenous Neighborhood Formation.

JEL Classification: C72, C73

*Sant'Anna School of Advanced Studies, P.zza Martiri della Libertà, 33, I-56127 PISA (Italy). Tel: +39-050-883-343. Fax: +39-050-883-344. Thanks to Giovanni Dosi, Karl Schlag, Alan Kirman, Sanjeev Goyal, and many participants to "The Society for Computational Economics" Conference (Aix-enProvence, France, 26-29 June 2002) for stimulating and helpful comments. 


\section{Introduction}

The outcome of a coordination game repeatedly played in large societies might be crucially affected by the coevolution between interaction structures and individual choices.

In the last years, this issue has been investigated in an evolutionary game setting by assuming exogenously fixed networks. In these models, agents are supposed to repeatedly play a $2 \times 2$ coordination game either against any other player in the population (cf. Kandori, Mailath \& Rob (1993) and Young (1996)) or against a fixed, typically small, set of 'relevant others' (see Blume (1993), Ellison (1993) and Young (1998)). The basic rationale is that, in many situations, the time-scale at which agents choose their opponents in the game is very long as compared to that at which they update their stage-game strategies. Consequently, it may be plausible to study the long-run properties of population learning as if interaction structures were static (cf. Skyrms \& Pemantle (2000)).

However, the evolution of individual choices through time might also affect agents' expectations about future payoffs from interactions. A player might then change her idea on who is in fact 'relevant' and avoid further interactions (or, respectively, start interacting) with groups of other agents if she expects that this would lead to a decrease (or, respectively, an increase) in net payoffs. In such circumstances, the speed at which agents revise the set of their partners is (at least) comparable to the frequency at which they update their strategy. Therefore, the details of the process governing the coevolution between strategies and interaction structures may have non-trivial consequences on long-run coordination patterns (see Kirman (1997) for a discussion).

To address this issue, we present here a dynamic model of coordination in which agents can simultaneously choose the strategy to play in the game and select the agents with whom to play the game. We consider a population of myopic individuals located on a circle who repeatedly play a pure coordination game with their 'nearest neighbors'. We assume that holding neighbors is costly and that, from time to time, agents are allowed to slightly shrink or enlarge the 'radius' of their current neighborhood by maximizing expected net payoffs. Local neighborhood adjustment reflects here the well documented inertia of many social relationships (see e.g. Miller (1963) and Akerlof (1997)). We focus on settings characterized by network externalities which may be positive when agents hold small neighborhoods sizes, but become eventually negative when neighborhood sizes are very large (e.g. due to network congestion effects).

After having characterized the set of steady states and conditions for convergence, we show that both full coordination and coexistence of conventions may be possible in the long-run. However, as computer simulations show, the system is able to robustly reach very high coordination levels together with small neighborhood sizes despite the presence of negative local network externalities. Moreover, we find that average coordination in 
presence of a non-zero (although small) frequency of neighborhood adjustment is higher than if interaction structures were static. Furthermore, we show that large societies better coordinate provided that initial average neighborhood sizes are sufficiently high. Finally, we test the robustness of the model to departures from the basic setup. In particular, we study what happens to long-run patterns of coordination when agents: (i) might enjoy positive network externalities for all neighborhood sizes; (ii) are located in higher-dimensional lattices; (iii) play coordination stage-games where a trade-off arises between Pareto-efficient and risk-dominant Nash equilibria.

The rest of the paper is organized as follows. In Section 2, we review related works and we discuss the extent to which our model departs from existing literature. In Section 3 we formally describe the model. Section 4 deals with the characterization of absorbing states in the basic setup, while computer simulation results on the average long-run behavior of the system are reported in Section 5. In Section 6 we discuss extensions of the basic setup. Finally, Section 7 concludes.

\section{Coordination and Endogenous Neighborhood For- mation}

The issue of coordination in presence of endogenous partner selection has been recently addressed by Goyal \& Vega-Redondo (2001), Jackson \& Watts (2000) and Droste, Gilles $\&$ Johnson (2000) in the framework of network formation ${ }^{1}$. These models share four basic assumptions. First, agents are able to simultaneously update stage-game strategies and directly choose whom to interact with.

Second, no underlying socio-economic geography prevents (or restricts) interactions between 'distant' agents. Players in Goyal \& Vega-Redondo (2001) and Jackson \& Watts (2000) can indeed form or delete single costly links with any other agent in the population on the basis of myopic expectations about the future net benefit from coordination in alternative network structures. On the contrary, Droste et al. (2000) assume players located on a circle and suppose that the probability of a link between any two agents decreases with their distance. Nevertheless, players can potentially observe the current strategy of any other agent in the population before choosing whether to establish a link with her or not, irrespective of the distance between them.

Third, the speed at which agents can change their partners is the same as the pace at which they update their strategy. In fact, everytime a player is called to revise her current

\footnotetext{
${ }^{1}$ See also Goyal \& Janssen (1997), Skyrms \& Pemantle (2000) and Mailath, Samuelson \& Shaked (2000). Dynamic models of non-cooperative network formation only (i.e. without simultaneous choice of a strategic variable) are studied in Bala \& Goyal (2000), Watts (2001) and Jackson \& Watts (1999).
} 
state, she can simultaneously change both her pure stage-game strategy and the set of partners (possibly in different stages of a same time period).

Finally, existing models study coordination and network formation in a positive network externalities setup. More specifically, they typically assume that the gross (i.e. before link costs) payoff to an agent playing a given strategy $s$ is not affected by the size of the network, provided that the number of connected players choosing $s$ does not change. If the cost of forming a link is sufficiently small, net payoffs always increase as the size of the network (i.e. connected players choosing the same strategy) increases. On the contrary, if unit link costs are very large negative network externalities arise for all network sizes ${ }^{2}$. Therefore, there are no network size effects on gross (and net) payoffs and the regime of network externalities depends on the level of unit link costs.

In this paper, we present a dynamic, non-cooperative, model of coordination and neighborhood formation in which strategies and interaction structures are both subject to endogenous evolution. As happens in Droste et al. (2000), we suppose that agents are spatially located on the nodes of a circle and play $2 \times 2$ pure coordination games with their nearest neighbors. However, in contrast with existing models, we assume that in each time period players are allowed either to update their current pure strategy (given the current interaction structure) or, with some exogenously fixed probability, to simultaneously adjust strategies and neighbors. This allows us to study situations in which neighborhood adjustment is endogenous but relatively not frequent.

We suppose that players act myopically and update pure strategies and/or neighbors by best-responding to their current environment in a completely deterministic way (i.e. without mistakes) ${ }^{3}$. Unlike Droste et al. (2000), we do not allow for 'jumps' in neighborhood adjustment. Indeed, we posit that agents update their neighbors' set by adding to (or discard from) it only those players who are located close to the boundaries of the neighborhood. More precisely, if an agent interacts at time $t-1$ with all individuals placed no farther than $r>1$ nodes from her, she will only consider to locally adapt the radius of her neighborhood, i.e. shrink it to $r-1$, enlarge it to $r+1$ or keep it unchanged, while simultaneously choosing whether to stick to her current strategy or not. As holding neighbors is assumed to be costly (total cost is proportional to neighborhood size), a player will simply adjust her current set of partners by comparing net payoffs from coordination.

In our model, the underlying geography strongly constrains the set of options available to any agent. In fact, the assumption that individuals cannot observe current choices

\footnotetext{
${ }^{2}$ This case is not however adressed in the literature. In fact, if agents have the option not to play the game, empty networks will always arise because agents will always prefer to hold empty neighborhoods.

${ }^{3}$ This contrasts with adaptive play with mistakes studied in Goyal \& Vega-Redondo (2001) and Jackson \& Watts (2000), while is in line with fair parts of Droste et al. (2000) and previous work by Blume (1993) in the context of static interaction structures.
} 
of agents placed far from them implies that the choice set is quite small ${ }^{4}$. Furthermore, in contrast with existing literature assuming agents who directly select their partners in a sequence of one-to-one link additions/deletions, we suppose that players can only indirectly choose whom to interact with by adjusting their 'interaction window'.

Finally, we study a setup where network externalities become eventually negative as neighborhood sizes increase. In particular, we assume that there exist (negative) neighborhood size effects on gross payoffs from interaction: an agent currently playing strategy $s$ who faces two neighborhoods characterized by the same number of partners choosing $s$ will earn a higher gross payoff if she chooses the smaller one. Therefore, given unit link costs, agents could be willing to enlarge their neighborhood size if the latter is relatively small, while they will eventually prefer to shrink it (no matter the local strategy configuration they face) if the number of neighbors goes beyond some endogenously determined threshold.

This property, together with the assumption that agents cannot refuse to play the game, allows us to address the issue of coordination in presence 'locally' positive but 'globally' negative network externalities. Indeed, in many circumstances well documented e.g. in the 'economics of the Internet' literature ${ }^{5}$, individual net payoffs might eventually fall as the network size increases irrespective of pure strategies played by the agents either: (i) because of congestion problems entailed by the physics of the network; or (ii) because the net added value of any new player is negligible to each individual belonging to the network. In particular, players may prefer to avoid unwanted interactions as unknown agents may entail risks (e.g. a higher likelihood of computer viruses). Moreover, whenever agents are status seeking and aspire to be similar to their peers, individuals might dislike coordination if the group to which they belong becomes too crowded (see e.g. Cowan, Cowan \& Swann (1998)).

We start by studying a basic setup where agents play a purely symmetric coordination game, i.e. a coordination game where both strategies are equivalent with respect to Pareto efficiency and risk-dominance. The goal is to study whether endogenous neighborhood formation alone has a significant impact on long-run coordination, in presence of spatiallylocated agents who locally adapt their neighborhoods and strategies ${ }^{6}$. In particular, we

\footnotetext{
${ }^{4}$ In Goyal \& Vega-Redondo (2001) agents can observe the entire strategy configuration and choose among all conceivable networks at any time. This presumes agents with huge computational skills and seems to contrast with myopic learning. Conversely, in Jackson \& Watts (2000) and Droste et al. (2000), agents can form at most one link per period, but they can potentially add to their set of partners any other agent in the population.

${ }^{5}$ See e.g. Mitchell (2000) and Gupta, Stahl \& Whinston (1995). For a more general perspective, cf. Economides (1996) and Arnott \& Small (1994).

${ }^{6}$ Droste et al. (2000) find coexistence of conventions to be possible in adaptive play without mistakes. However, their model does not deliver any prediction about the level of aggregate coordination in such equilibria (e.g. the average number of coordinated players) and about how likely coexistence of convention is (e.g. as the parameters of the model change).
} 
will analyze the extent to which allowing for simultaneous adjustment of players' strategies and neighbors results in higher long-run average coordination, as compared to cases in which neighborhood structures are static. Furthermore, we explore how coordination and interaction structures are affected by: (i) the speed of neighborhood adjustment; (ii) unit neighborhood costs; (iii) the strength of negative network externalities. Some interesting departures from the basic setup are instead discussed in Section 6.

\section{The Model}

Consider a finite set of agents $I=\{1,2, \ldots, N\}, N \geq 5$. Agents are spatially distributed on the nodes of a 1-dimensional lattice with periodic boundaries (i.e. a circle). We assume a one-to-one relation between the nodes of the lattice and the set of agents. Hence, individual labels $i=1,2, \ldots, N$ coincide with locations on the circle. Time is discrete. At each $t=1,2, \ldots$ any agent plays a pure $2 \times 2$ coordination game against her current partners. More precisely, if agent $i$ plays $s_{i} \in\{-1,1\}$ when agent $j^{\prime} s$ strategy is $s_{j} \in\{-1,1\}$, her stage-game payoff is given by:

$$
G\left(s_{i}, s_{j}\right)=\left\{\begin{array}{lll}
1 & \text { if } & s_{i}=s_{j} \\
0 & \text { if } & s_{i} \neq s_{j}
\end{array} .\right.
$$

Any agent $i$ is completely characterized by her set of partners $V_{i t} \subset I$ and her current strategy $s_{i t} \in S=\{-1,+1\}$. Following Ellison (1993), we assume that agent $i$ 's partners set consists of all players located within a neighborhood of radius $r_{i t}$. More formally:

$$
V_{i t}=V\left(r_{i t}\right)=:\left\{j \in I: 0<\min \{|j-i|, N-|j-i|\} \leq r_{i t}\right\}
$$

where $r_{i t} \in R(N)=\{1,2, \ldots, \widetilde{r}\}$ and $\widetilde{r}=\lfloor N / 2\rfloor$. As $V_{i t}$ only depend on $r_{i t}$, we define the state of any agent $i$ at time $t$ as:

$$
\left(s_{i t}, r_{i t}\right) \in S \times R(N) .
$$

The economy will be in turn characterized at any $t$ by its global configuration:

$$
\Omega_{t}=\left(s_{i t}, r_{i t}\right)_{i \in I}
$$

where $\Omega_{t} \in \Gamma(N)=(S \times R(N))^{N}$.

In each time period, an agent whose state is $\left(s_{i t}, r_{i t}\right)$ will earn a (net) overall payoff 
defined as:

$$
\pi_{i t}\left(s_{i t} ; r_{i t} \mid \Omega_{t}\right)=\left[\frac{1}{\left|V\left(r_{i t}\right)\right|^{\alpha}} \sum_{j \in V\left(r_{i t}\right)} G\left(s_{i t}, s_{j t}\right)\right]-\beta\left|V\left(r_{i t}\right)\right|
$$

where $G\left(s_{i t}, s_{j t}\right)$ is defined in (1), $\left|V\left(r_{i t}\right)\right|$ is the size of $V\left(r_{i t}\right)$ and $\beta>0$ is the unit cost per neighbor (i.e. the cost of keeping active at $t$ the link to any agent $j \neq i$ ).

Notice that the parameter $\alpha \in[0,1]$ governs the nature of local network externalities in the model. To see why, let us consider two extreme cases. On the one hand, if $\alpha=0$, there are no neighborhood size effects on gross payoffs (i.e. the term in square brackets): if the number of agents playing $s_{i t}$ in $V\left(r^{\prime}\right)$ and $V\left(r^{\prime \prime}\right)$ is the same, then gross payoffs do not change. Therefore, if $\beta>1$ the system is characterized by 'always negative' network externalities because net payoffs are strictly decreasing with $r_{i t}$ (and thus with the number of agents playing $s_{i t}$ in $V$ ) irrespective of strategy configuration. Conversely, if $\beta<1$ (and sufficiently small) network externalities are 'always positive': net payoffs are strictly increasing in the size of the network (i.e. in the number of players choosing $s_{i t}$ in $\left.V\left(r_{i t}\right)\right)^{7}$.

On the other hand, if $\alpha=1$, gross payoffs from playing $\left(s_{i t}, r_{i t}\right)$ are negatively affected by the size of the neighborhood. For a given strategy configuration, net payoffs might increase if neighborhood sizes are not too large, because gross gains from local coordination can offset costs. However, they will eventually fall, irrespective of currently played strategies, as neighborhood size grows. In this case the system is potentially characterized by 'locally' positive and 'globally' negative network externalities.

In this paper, we will primarily focus on the latter case $(\alpha=1)$. The behavior of the system when $\alpha \in[0,1)$ is explored in Section 6.1. When $\alpha=1$, as $\left|V\left(r_{i t}\right)\right|=2 r_{i t}$ and $G\left(s_{i t}, s_{j t}\right)=1$ if and only if $s_{i t}=s_{j t}$, payoffs in (3) read:

$$
\pi_{i t}\left(s_{i t} ; r_{i t} \mid \Omega_{t}\right)=\left\{\begin{array}{ccc}
x_{i}\left(r_{i t}\right)-\gamma r & \text { if } & s_{i t}=+1 \\
1-x_{i}\left(r_{i t}\right)-\gamma r & \text { if } & s_{i t}=-1
\end{array},\right.
$$

where $\gamma=2 \beta$ ('unit cost', in the following) and $x_{i}\left(r_{i t}\right) \in[0,1]$ is the share of agents in $V\left(r_{i t}\right)$ currently playing +1 ('local frequency' in the following).

Let us turn now to describe the dynamics of the model. At the beginning of period $t+1$ an agent (say $i$ ) is drawn at random from $I$. With probability $1-\theta \in(0,1]$ she is allowed to update her current strategy $s_{i t}$ only (given $r_{i t}$ ), while with probability $\theta \in(0,1]$ she can simultaneously update both $s_{i t}$ and $r_{i t}{ }^{8}$.

\footnotetext{
${ }^{7}$ Strictly speaking, the regime of network externalities (positive vs. negative) is not equivalent to strict monotonocity (increasing or decreasing) of net payoffs with respect to neighborhood size. Since agents do not directly select their partners, a higher $r$ might indeed entail a non-increasing number of coordinated agents.

${ }^{8}$ All results presented in the next Sections are not qualitatively altered if one instead assumes that,
} 
Consider first strategy updating only. We assume that agent $i$ best-responds (without mistakes) to the current local configuration. Thus:

$$
s_{i, t+1} \in \underset{s \in\{-1,+1\}}{\arg \max } \pi_{i t}\left(s ; r_{i t} \mid \Omega_{t}\right) .
$$

We also suppose that agents are change-averse, i.e. they stick to their current choice when ties occur.

On the contrary, an agent drawn to update both $s_{i t}$ and $r_{i t}$ will only be able to locally adjust her neighborhood radius and her current strategy. More formally, we suppose that given $\left(r_{i t}, s_{i t}\right.$ ), agent $i$ will only have (for both $s=s_{i t}$ and $s=-s_{i t}$ ) the following three options: (i) shrinking to $r_{i t}-1$; (ii) keeping $r_{i t}$; or (iii) enlarging to $r_{i t}+1$. Agents myopically choose the best-response among their available options, i.e.:

$$
\begin{aligned}
\left(s_{i, t+1}, r_{i, t+1}\right) \in \arg \max & \pi_{i t}\left(s ; r \mid \Omega_{t}\right), \\
& \text { s.t. } \quad s \in\{-1,1\} \\
& \quad r \in R(N):\left|r-r_{i t}\right| \leq 1
\end{aligned} .
$$

As happens in action updating, we suppose that players are change-averse. Furthermore, we assume for the sake of simplicity that, whenever $\left(s, r_{i, t}+1\right)$ and $\left(s, r_{i, t}-1\right)$ are the unique solutions of $(6)$ for $s \in\{-1,1\}$, agents always choose $\left(s, r_{i, t}-1\right)^{9}$.

Given any initial conditions $\Omega_{0} \in \Gamma(N)$, eqs. (2), (4), (5) and (6) define a Markov chain $\left\{\Omega_{t}, t \geq 1\right\}$ with finite state-space $\Gamma(N)$.

In the following section, we will characterize the set of steady states of the dynamic process (i.e. absorbing states of the Markov chain). We will show that this set is non empty and that the system almost always converges to a steady state in finite time. Moreover, we will characterize steady-states in terms of aggregate coordination and neighborhood structures. We will show that both full coordination and coexistence of conventions can arise in a SS. Furthermore, we will provide examples of SS in which coexistence of conventions arises together with heterogenous neighborhood structures.

in each period $t$, an agent currently playing $\left(s_{i, t}, r_{i, t}\right)$ is allowed with probability $\theta \in(0,1)$ to revise only her current neighborhood size given the current strategy configuration (i.e. she is not allowed to change strategy and radius at the same time).

${ }^{9}$ This assumption does not crucially affect the dynamic properties of the system. In fact, one might equally employ the alternative assumption (i.e. always choose $\left(s, r_{i, t}+1\right)$ ) without loss of generality. Moreover, as shown in Appendix A, this tie-break case can only arise if unit cost $\gamma$ lies in a non-dense subset of $R_{+}$(i.e. for residual choices of $\gamma$ ). In what follows, we will not employ randomization (i.e. choose with equal probability between $\left(s, r_{i, t}-1\right)$ and $\left.\left(s, r_{i, t}+1\right)\right)$ to simplify the analysis of convergence (cf. Prop. 2 below). 


\section{Steady States, Convergence and Coordination}

A steady-state (SS) of the system (i.e. an absorbing state for $\left\{\Omega_{t}, t \geq 1\right\}$ ) is defined as a global configuration $\Omega^{*}=\left\{\left(s_{i}^{*}, r_{i}^{*}\right), i \in I\right\}$ such that any player $i \in I$ has no incentive to unilaterally move away from $\left(s_{i}^{*}, r_{i}^{*}\right)$. A SS is formally characterized as follows:

Lemma 1 (Characterization of Steady States) Denote by $x_{i, r_{i}^{*}}^{*}=x_{i}\left(r_{i}^{*}\right)$ the frequency of players choosing +1 in the neighborhood of agent $i$ when the global configuration is $\Omega^{*}$. Then $\Omega^{*}$ is a $S S$ for $\left\{\Omega_{t}, t \geq 1\right\}$ if and only if:

1. For any $i \in I$ s.t. $s_{i}^{*}=+1$ :

$$
\begin{array}{ll}
r_{i}^{*}=1: & x_{i, r_{i}^{*}}^{*} \geq \max \left\{-\gamma+x_{i, 2}^{*}, \frac{1}{2},-\gamma+1-x_{i, 2}^{*}\right\} \\
1<r_{i}^{*}<\widetilde{r}: & x_{i, r_{i}^{*}}^{*} \geq \max \left\{\gamma+x_{i, r_{i}^{*}-1}^{*}, \gamma+1-x_{i, r_{i}^{*}-1}^{*}, \frac{1}{2},-\gamma+x_{i, r_{i}^{*}+1}^{*},-\gamma+1-x_{i, r_{i}^{*}+1}^{*}\right\} \\
r_{i}^{*}=\widetilde{r}: & x_{i, r_{i}^{*}}^{*} \geq \max \left\{\gamma+x_{i, \widetilde{r}-1}^{*}, \frac{1}{2}, \gamma+1-x_{i, \widetilde{r}-1}^{*}\right\}
\end{array}
$$

2. For any $i \in I$ s.t. $s_{i}^{*}=-1$, the lhs in the above inequalities are satisfied if $x_{i, r_{i}^{*}}$ is replaced by $\left(1-x_{i, r_{i}^{*}}^{*}\right)$.

Proof. Since $\theta>0$, if $\left(s_{i}^{*}, r_{i}^{*}\right)$ is stable with respect to neighborhood updating, it will be also stable for strategy updating. Hence, it suffices to impose the condition $\left(s_{i, t+1}, r_{i, t+1}\right)=$ $\left(s_{i}^{*}, r_{i}^{*}\right)$ in Eq. (6) and solve for $x_{i}\left(r_{i}^{*}\right)$.

Consider now the set $\Gamma^{*}(\gamma, N)$ of all $\Omega^{*} \in \Gamma(N)$ satisfying SS conditions. Notice that $\Gamma^{*}(\gamma, N)$ does not depend on $\theta$, as the speed of strategy/action updating does not influence updating rules. Furthermore, for any $(\gamma, N), \Gamma^{*}(\gamma, N)$ is non-empty as it always contains (at least) both configurations $\Omega^{1}=(1,1)_{i \in I}$ and $\Omega^{-1}=(-1,1)_{i \in I}$. Indeed, if all agents choose the same strategy then $x_{i}(1) \in\{0,1\}$ for all $i$. Hence, as it can be easily seen by replacing $x_{i, r_{i}^{*}}$ with either 0 or 1 in SS conditions, no agent has an incentive to enlarge her current radius.

In Proposition 2, we show that in the long run the process $\left\{\Omega_{t}, t \geq 1\right\}$ will almost always converge in finite time to a point of $\Gamma^{*}(\gamma, N)$ for all $(\theta, \gamma, N)$. To see why, notice that in each time period one and only one agent can update her current state by best-replying to local strategies profiles. Moreover, tie-breaking rules defined in Section 3 imply that individual best-replies are unique given the current configuration. Hence, the resulting best-reply strategy revision process governing the evolution of configurations $\Omega$ is characterized by the so-called 'unique best-reply property' (see Blume (1995)). For such class of Markov processes, we know that there is a one-to-one correspondence between absorbing states 
and ergodic sets (i.e. all configurations which are not SS are transient). Hence, finite-time convergence to an absorbing state is a probability one event in our system.

Proposition 2 (Convergence to SS) For any given $N \geq 5, \theta \in(0,1], \gamma>0$ and $\Omega_{0} \in$ $\Gamma(N)$, the process $\left\{\Omega_{t}, t \geq 1\right\}$ defined by eqs. (2), (4), (5) and (6) will almost always converge in finite time to an absorbing state $\Omega^{*} \in \Gamma^{*}(\gamma, N)$.

\section{Proof. See Appendix A.}

As typically happens in best-response strategy revision games without trembles, the set $\Gamma^{*}(\gamma, N)$ is likely to contain many other absorbing states apart from the ones characterized by full coordination configurations with minimal neighborhood size. In Proposition 3, we start characterizing the set of SS. In particular we ask whether (and in which parameters region) the system is able to sustain SS involving high vs. low levels of coordination, together with homogeneous vs. heterogeneous neighborhood structures.

Proposition 3 Consider a $S S \Omega^{*} \in \Gamma^{*}(\gamma, N)$ and define $\widehat{s}=N^{-1} \sum_{i=1}^{N} s_{i}^{*}$. Then:

1. For any $(\gamma, N)$, there exist $S S$ in which $\widehat{s} \in\{-1,1\}$, i.e. full coordination arises. Such SS necessarily imply $r_{i}^{*}=1, \forall i \in I$ (neighborhood homogeneity with smallest neighborhood sizes).

2. For any $(\gamma, N)$, there exist SS in which $\widehat{s} \in(-1,1)$, i.e. coexistence of conventions arises.

3. For any $(\gamma, N)$, coexistence of conventions and homogeneity of neighborhood sizes simultaneously arise in a SS only if $r_{i}^{*}=1 \forall i \in I$.

4. There exist parameter values $(\gamma, N)$ such that in a $S S$ coexistence of conventions and heterogeneous neighborhood sizes is possible. More precisely, heterogeneous strategy configurations can sustain heterogeneous neighborhood sizes (for a given population size) only if $\gamma \leq \frac{1}{N-3}$.

\section{Proof. See Appendix A.}

The main finding is that both full and partial coordination can in principle arise as a SS. However, unlike what happens in Goyal \& Vega-Redondo (2001), Jackson \& Watts (2000) and Droste et al. (2000), negative network externalities prevents any network different from the minimally connected one (and thus also the complete one) to sustain full coordination in a SS, no matter how small unit costs are. This is because full coordination implies that 
every agent gets the highest possible gross payoff irrespective of her neighborhood size, so that enlarging the neighborhood necessarily leads to smaller payoffs.

Conversely, strategy configurations characterized by low coordination can arise together with both homogeneous (with radius 1) and heterogeneous interaction structures. As illustrated in the following example, the same heterogeneous strategy configuration can in principle sustain (for sufficiently small $\gamma$ ) many alternative interaction structures entailing different aggregate interaction costs for the society.

Example 4 Suppose that $N=11$ (i.e. $\widetilde{r}=5$ ) and that the following strategy configuration is given:

$$
s_{i}^{*}=\left\{\begin{array}{cc}
+1 & i=1, \ldots, 8 \\
-1 & i=9,10,11
\end{array} .\right.
$$

Consider now the two following interaction structures: $(A): r_{i}^{*}=1, i=1, \ldots, 11$; and $(B): r_{i}^{*}=1, i \neq 7,8 ; r_{i}^{*}=5, i=7$, 8. If $\gamma \leq 3 / 40$, then both $(A)$ and $(B)$, together with $(*)$, are $S S$, while if $\gamma>3 / 40$ only $(B)$, together with $(*)$, can be a $S S$.

Proof. It suffices to check that the state $\left(s_{i}^{*}, r_{i}^{*}\right)$ of any agent $i$ is locally stable. See Lemma 5 in Appendix A.

Notice that in the example the first configuration is homogenous and allows the system to reach the lowest aggregate neighborhood cost $(N \gamma)$, while the second one is heterogeneous and inefficient, because aggregate neighborhood cost is strictly larger than $N \gamma$.

Yet, a heterogeneous strategy configuration can sustain a homogeneous neighborhood structure only if neighborhood size is the minimal one. This is because the larger neighborhood sizes held by two adjacent agents who play opposite strategies, the larger the number of common neighbors playing +1 who are needed in order for that configuration to be locally stable with respect to strategy updating (see App. C). This prevents sustainability of interaction structures with higher neighborhood sizes (e.g. with $r>1$ all $i$ ).

Furthermore, heterogeneous strategy configurations cannot sustain heterogeneous radii for all parameter values $(\gamma, N)$. As local network externalities become eventually negative when neighborhood sizes increase for a given $N$, any agent holding a $r$-neighborhood faces stronger negative network externalities effects as $\gamma$ increases given $r$, or, equivalently, as $r$ increases given $\gamma$. In the extreme case, it is possible to show (see App. B) that if $\gamma(r-1)>\frac{1}{2}$ and $r \geq 2$, the agent will always shrink to $r-1$ (and simultaneously switch strategy) irrespective of the local strategy profile. 
Therefore, heterogeneous strategy configurations can sustain heterogeneous radii only if $\gamma$ is sufficiently small for a given population size. In such situations agents trade-off between positive and negative network externalities. On the one hand, players try to adjust strategy and neighborhood so as to increase gross payoffs given their local environment. If network externalities are perceived to be positive even at large distances (e.g. strategy configuration is sufficiently heterogeneous), some agents have the incentive to enlarge their information 'window' up to values close to $\widetilde{r}$. On the other hand, as $\gamma$ increases for given $N$, negative network externalities tend to dominate even for small neighborhood sizes. In such a situation, agents always shrink their radius while simultaneously switching strategies up to the point at which all agents will hold $r=1$.

So far, we attempted to provide a characterization of the SS of the system as far as heterogeneity vs. homogeneity of strategies and neighborhood structures are concerned. In the next Section, we will turn to Montecarlo (MC) computer-simulations exercises to shed light on the long-run behavior of the system. In particular, we will explore how system parameters affect long-run coordination and interaction structures.

\section{The Long-Run Behavior of the System: Simulation Results}

Let $M$ be the number of realizations of the stochastic process $\left\{\Omega_{t}, t \geq 1\right\}$ generated from a sample of random, independently drawn, initial conditions $\left\{\Omega_{0, m} \in \Gamma, m=1, \ldots, M\right\}$. Since for any choice of $(\gamma, \theta, N)$ convergence to a $\operatorname{SS} \Omega_{m}^{*}=\left(s_{i, m}^{*}, r_{i, m}^{*}\right)_{i=1}^{N}$ is a probability-one event in each run $m$, we can study the long-run behavior of the system by analyzing MC distributions of some interesting statistics computed on $\Omega_{m}^{*}$.

More specifically, let us define average SS coordination by $\bar{c}_{m}=\left|\bar{s}_{m}\right|=\left|N^{-1} \sum_{i=1}^{N} s_{i, m}^{*}\right|$ and, accordingly, SS average neighborhood size (i.e. average radius) by $\bar{r}_{m}=N^{-1} \sum_{i=1}^{N} r_{i, m}^{*} \in$ $[1, \widetilde{r}]$. In the following, we will explore how long-run coordination and neighborhood patterns depend on system parameters by focusing on how $(\widehat{c}, \widehat{r})=\left(M^{-1} \sum_{i=1}^{M} \bar{c}_{m}, M^{-1} \sum_{i=1}^{M} \bar{r}_{m}\right)$ change with $(N, \gamma, \theta)^{10}$.

\subsection{Coordination and Neighborhood Formation}

A first clear-cut result that $\mathrm{MC}$ simulations point out is that, for any $(N, \gamma, \theta)$, the likelihood of absorption in SS characterized by high average coordination and small average

\footnotetext{
${ }^{10}$ The MC sample size $M$ is usually chosen between 1000 and 10000 . In general, this range is sufficient both to attain a good precision of the estimates (e.g. extremely low MC variances $\sigma^{2}\left(\bar{c}_{m}\right)$ and $\sigma^{2}\left(\bar{r}_{m}\right)$ ) and, at the same time, to keep the probability of duplications in drawing initial configurations below $0.5 \%$, even for small $N$. Since MC variances do not change very much across the parameter space, we can neglect the study of coefficients $\bar{c}_{m} / \sigma^{2}\left(\bar{c}_{m}\right)$ and $\bar{r}_{m} / \sigma^{2}\left(\bar{r}_{m}\right)$ and focus on MC means only.
} 
neighborhood sizes is very large. More precisely:

Observation 1: In $84.6 \%$ of runs, the society is absorbed in SS characterized by average coordination levels $\bar{c}_{m} \geq 0.9$. Moreover, in $89.6 \%$ of cases the system is absorbed either by $\Omega^{1}=(1,1)_{i \in I}$ or $\Omega^{1}=(-1,1)_{i \in I}$, while in $99.1 \%$ of runs the scaled average radius $\bar{R}_{m}=\widetilde{r}^{-1} \bar{r}_{m} \in(0,1]$ is smaller than 0.50 .

Despite the presence of negative local network externalities, the society seems therefore able to exploit neighborhood adjustment to reach configurations that are globally characterized by high levels of coordination. Nevertheless, as one starts exploring the long-run behavior of the system as $(\gamma, \theta)$ vary in the relevant parameter space for a given population size $N$, it appears that the system may be trapped, with a small but significant likelihood, in long-run configurations with low average coordination. In Figs. 1a and 1b, we depict an example of how $\widehat{c}$ and $\widehat{r}$ change with $(\gamma, \theta)$ for $N=21$. Aggregate coordination, as measured by $\widehat{c}$, attains values close to $70 \%$ when both $\gamma$ and $\theta$ are large. In the same region of the parameter space, the frequency of $\mathrm{MC}$ simulations in which the system is absorbed either by $\Omega_{+1}^{*}$ or by $\Omega_{-1}^{*}$ is around $50 \%$. Conversely, average neighborhood sizes, while staying very close to 1 for almost all sampled $\gamma$ 's and $\theta$ 's, seem to decrease with unit costs and, at least when the latter are small, to weakly increase with the frequency of neighborhood adjustment. In general, the evidence about how $\gamma$ and $\theta$ affect coordination and interaction structures for any given $N$ can be summarized in the following observations.

Observation 2: For all $N>4$, average coordination $\widehat{c}$ is always non-increasing in $\gamma$ and $\theta$. In particular, the frequency of all runs in which $\bar{c}_{m}>0.95$ is 0.48 when $\gamma \geq 0.5$ and 0.74 when $\gamma<0.5$. Moreover, the frequency of all runs in which $\bar{c}_{m}>0.95$ is 0.54 when $\theta \geq 0.1$ and 0.77 when $\theta<0.1$.

Observation 3: For all $N>4$, average neighborhood sizes $\widehat{r}$ is always non-increasing in $\gamma$ and non-decreasing in $\theta$. In particular, the frequency of all runs in which $\bar{r}_{m}>1$ is 0.077 when $\gamma \geq 0.5$ and 0.066 when $\gamma<0.5$. Moreover, the frequency of all runs in which $\bar{r}_{m}>1$ is 0.088 when $\theta \geq 0.1$ and 0.065 when $\theta<0.1$.

Therefore, smaller unit costs imply higher average neighborhood sizes together with higher aggregate coordination, while a lower speed of neighborhood adjustment induces higher aggregate coordination. Figs. (2a-2d) plot the typical time evolution of $\bar{c}_{m}$ and $\bar{R}_{m}$ in four setups characterized by small (resp. large) unit costs and small (resp. large) speed of neighborhood adjustment. Recall that given $N$, a larger $\gamma$ implies stronger negative externalities network effects (even locally), and possibly agents willing to shrink their neighborhoods no matter strategy configurations. Conversely, a small $\gamma$ induces larger flexibility in local payoff improvements through the exploitation of positive local network 
externalities. Moreover, a small speed of neighborhood updating allows players to better coordinate with their neighbors given the current interaction structure, especially when the latter is characterized by large neighborhoods (as typically happens at the beginning of the realizations).

Hence, when $\gamma$ is very small, agents are expected to employ neighborhood updating to locally coordinate with each other. This would in turn lead to an initial increase of $\bar{c}_{m}$ in response of fluctuations in $\bar{r}_{m}$. However, whether full aggregate coordination will be attained or not depends on the magnitude of $\theta$. If $\theta$ is small (cf. Fig. 2a), agents are able to fully exploit their currently large neighborhoods to better coordinate. Thus $\bar{c}_{m}$ quickly converges to 1 and, from then on, $\bar{r}_{m}$ slowly decreases toward 1 (i.e. $\bar{R}_{m}$ tends to $\left.\widetilde{r}^{-1}\right)$. Conversely, when $\theta$ is large, the society can sometimes be trapped in a low coordination configuration that sustains higher average neighborhood sizes (see Fig. $2 b)$. On the contrary, if $\gamma$ is very large, negative network externalities induce agents to minimize costs by shrinking neighborhood sizes irrespective of gross payoffs. Hence, $\bar{r}_{m}$ steadily decreases from the beginning of the process. This introduces turbulence in strategy configurations and makes harder to reach full coordination, because agents always switch their strategies while shrinking (see App. B). Once again, whether full or partial coordination will be achieved strongly depends on the speed of neighborhood adjustment. In general, the smaller $\theta$, the quicker the system will converge to $\bar{c}_{m}=1$ (see Figs. 2c and $2 \mathrm{~d}$ ). Here, however, SS configurations with $\bar{r}_{m}>1$ will be hardly reached, because $\gamma$ is too large to sustain neighborhood radii larger than 1.

Let us now turn to explore the effects of increasing the population size $N$ for given $(\gamma, \theta)$. The following observations summarize the results stemming from MC experiments.

Observation 4: If the initial neighborhood structure is drawn at random from $R(N)=$ $\{1,2, \ldots, \widetilde{r}\}$, then for all $N>4$ :

1. Average coordination $\widehat{c}$ is non-decreasing in $N$ and converges to 1 as $N \rightarrow \infty$ for all $(\gamma, \theta)$. More precisely, if $N \geq 100$ then $\widehat{c}>0.99$ in $92 \%$ of simulations.

2. The re-scaled MC average radius neighborhood size $\widehat{R}=M^{-1} \sum_{m} \bar{R}_{m}$ is non-increasing in $N$ and converges to 0 as $N \rightarrow \infty$, for all $(\gamma, \theta)$. More precisely, if $N \geq 100$ then $\widehat{R}<0.05$ in $99 \%$ of simulations.

Observation 5: If $R(N)=R=\{1,2, \ldots, \widetilde{R}\}$, where $\widetilde{R}$ is a constant, then average coordination $\widehat{c}$ tend to fall with population size for any $(\gamma, \theta)$ and $\widetilde{R}$. If $\widetilde{R} \leq 3$, average coordination $\widehat{c}$ is grater than 0.75 in $88 \%$ of simulations when $N \leq 11$, while, for all $N>11, \widehat{c}$ is grater than 0.75 in only $5 \%$ of runs. 
The foregoing results are not surprising if we recall that a larger population size implies a larger average for the distribution of initial neighborhood sizes, as $\widetilde{r}=\lfloor N / 2\rfloor$. This has two effects. First, for any given $\gamma$ (and if we consider wlog $N$ odd), there exists a threshold $r^{*}(\gamma)=(1+2 \gamma) / 2 \gamma$, independent of $N$, such that any agent holding neighborhoods with $r>$ $r^{*}(\gamma)$ will always shrink to $r-1$ irrespective of local frequencies. Therefore, as $N$ grows, the range of attainable radii increases and thus negative network externalities are more likely. Second, as discussed in App. C, heterogeneous SS strategy configurations tend to be less and less sustainable as the average neighborhood size increases (no matter if $\theta>0$ or not). Consequently, full coordination will be almost always the case for very large population sizes when $\widetilde{r}=\lfloor N / 2\rfloor$, as individuals can take advantage of larger overlaps in initial neighborhood structure. In such situation, the network of links channelling information among players is initially more connected. This speeds up individual learning and favors more coordinated configurations (cf. Fig. 3a).

On the contrary, when the ratio between the upper bound of the support of the initial radius distribution and the population size (e.g. $\widetilde{R} / N$ ) decreases with $N$, players are not able to fully exploit neighborhood learning to better coordinate. Therefore, heterogeneous strategy configurations are more easily attainable (cf. Fig. 3b).

Finally, notice that if $R(N)=\{1,2, \ldots, \widetilde{r}\}$, the relationship between population size and average neighborhood sizes $\widehat{r}$ depends on the trade-off between the expected increase in $\widehat{r}$ (due to a larger $\widetilde{r}$ ) and the strength of negative local network externalities. Hence, the smaller $\gamma$, the easier an increase of $\widehat{r}$ for small population sizes. However, when $N$ becomes sufficiently large, negative network externalities tend to offset any increase in average neighborhood size. Thus, $\widehat{r}$ will eventually fall and its maximum (as well as is maximand) will typically decrease with $\gamma$ (see Fig. 3c). As a result, the re-scaled average radius $\widehat{R}$ will always be non-increasing in population size.

\subsection{Static vs. Evolving Interaction Structures}

In the foregoing analysis, we have analyzed the average long-run behavior of the model when players can learn how to select their partners in order to locally coordinate with them. Despite the presence of negative network externalities, the system seems able to reach very high (although not necessarily full) coordination even in small societies. This is in particular true whenever: (i) the frequency at which players can simultaneously adjust strategy and neighbors is positive and small; (ii) neighborhood costs are small.

In this section we will address the issue of how average coordination levels attained by a system with positive probability of neighborhood updating (denoted by $\widehat{c}_{\theta>0}$ ) compares with those reached by a system in which interaction structures are static (i.e. $\widehat{c}_{\theta=0}$ ).

As a benchmark case, let us start by assuming that initial interaction structures are 
homogenous among agents. Notice that when $\theta=0$, the neighborhood structure is exactly the same studied in the existing literature on local coordination games (cf. e.g. Ellison (1993)). On the contrary, when $\theta>0$ we will allow agents to adjust strategies and radii starting from an initial interaction structure where all agents hold the same neighborhood size. In this case, the main results are the following.

Observation 6: Suppose that, for $\theta \geq 0$, the initial radius configuration is homogeneous, i.e. $r_{i 0}=r$ all $i \in I, r=1,2, \ldots, \widetilde{r}$. Then:

1. Average coordination with static neighborhoods is increasing in $r=1,2, \ldots, \widetilde{r}$ for any $N$ and converges to 1 as $r \rightarrow \widetilde{r}$. Specifically, if $\left(\widetilde{r}^{-1} r\right)<0.9, \widehat{c}_{\theta=0}$ is lower than 0.5 in $99 \%$ of runs. On the contrary, when $\left(\widetilde{r}^{-1} r\right)<0.9, \widehat{c}_{\theta>0}$ (i.e. average coordination with evolving networks) is greater than 0.73 in $99 \%$ of runs.

2. For any choice of $r=1,2, \ldots, \widetilde{r}$ such that the scaled radius $\widetilde{r}^{-1} r$ goes to zero as $N \rightarrow \infty, \widehat{c}_{\theta=0}$ is decreasing in $N$ and converges to 0 as $N \rightarrow \infty$. For the same parametrizations, a system with $\theta>0$ attains a SS average coordination level $\widehat{c}_{\theta>0}$ which is greater than $\widehat{c}_{\theta=0}$ in $95 \%$ of runs.

Therefore, a positive probability of neighborhood adjustment favors higher coordination levels, as compared to those attained with static homogeneous structures. In fact, as $r$ tends to $\widetilde{r}$ for a given $N$ and agents cannot adjust neighborhoods, the communication network tends to the complete one and thus $\widehat{c}_{\theta=0}$ increases. As Fig. 4a shows for the case $N=11$, $\widehat{c}_{\theta=0}$ is very small when $r \simeq 1$ and monotonically converges to 1 . Nevertheless, coordination levels attained in a system with the same homogeneous initial radius configuration and a positive rate of neighborhood adjustment are higher than those attained for $\theta=0$, for a wide range of system parameters.

Moreover, $\widehat{c}_{\theta=0}$ typically falls as $N$ increases when neighborhood radius is sufficiently small with respect to $N$, as the constraints induced on local frequencies by heterogeneous strategy configurations are increasingly less sustainable at a global level (see Fig. 4b for an illustration). If we allow for neighborhood adjustment $(\theta>0)$ and we force initial neighborhood radii to be homogeneous, average coordination always ends up to be larger than $\widehat{c}_{\theta=0}$. Once again, the combined effects of endogenous neighborhood formation and negative local network externalities sustain better long-run coordination patterns through the exploitation of favorable local improvements.

Finally, notice that similar results can be obtained when we assume heterogeneous initial interaction structures $\left\{r_{i 0}, i \in I\right\}$ (i.e. where $\exists i \in I$ and $j \in I, j \neq i$ such that $r_{i 0} \neq r_{j 0}$ ) for $\theta \geq 0$. Hence, if players can respond to their local environment by simultaneously adjusting both strategies and partners, the society seems able to attain better long-run average coordination patterns than in the case where interaction structures are static. 


\section{Beyond the Basic Setup: Some Preliminary Exer- cises}

In the foregoing Sections we have analyzed the dynamic properties of a quite simple coordination game played by a population of spatially-located, myopic, agents who can repeatedly update both the action to play in the game and the size of their interaction neighborhood. We have deliberately restricted our attention to a simple dynamic population game where: (i) agents are located on a one-dimensional lattice with periodic boundaries; (ii) the underlying stage-game is a pure-coordination one; (iii) network externalities are locally positive but globally negative (i.e. $\alpha=1$ in Eq. (3)). In this Section, we will try to provide some intuitions about the behavior of the system when one gradually departs from these assumptions.

\subsection{Positive vs. Negative Network Externalities}

To begin with, suppose that in Eq. (3) $\alpha=0$. In this case, an agent $i$ holding a radius $r_{i t}$ earns a net payoff:

$$
\pi_{i t}\left(s_{i t} ; r_{i t} \mid \Omega_{t}\right)=\left\{\begin{array}{ccc}
2 r_{i t}\left[x_{i}\left(r_{i t}\right)-\beta\right] & \text { if } & s_{i t}=+1 \\
2 r_{i t}\left[1-x_{i}\left(r_{i t}\right)-\beta\right] & \text { if } & s_{i t}=-1
\end{array} .\right.
$$

Notice first that if $\beta \geq 1$ net payoffs are always less (or equal) than zero for all $x_{i}\left(r_{i t}\right)$. Network externalities are always negative and agents will prefer to hold the smallest radius as possible $(r=1)$. Suppose instead that $\beta<1$. It is easy to see ${ }^{11}$ that if $\beta<\frac{1}{4}$ an agent will amost always enlarge her radius. For unit costs in this range, net payoffs are strictly increasing in the size of the network (i.e. the number of agents in the neighborhood playing +1 ) for almost all local configurations. Finally, if $\frac{1}{4} \leq \beta<1$, the regime of network externalities is mixed because neighborhood updating strongly depends on local frequencies.

By developing an argument similar to that in Proposition 3 (cf. Appendix A), one can easily characterize the SS of the process. On the one hand, it is possible to show that in a SS full coordination (i.e. $s_{i}^{*}=+1$ all $i$ or $s_{i}^{*}=+1$ all $i$ ) can sustain, for any $(N, \theta)$, only one of two alternative neighborhood structures: (i) the minimally connected one, i.e. $\left\{r_{i}^{*}=1, i \in I\right\}$, when $\beta>1$; or (ii) the complete network one, i.e. $\left\{r_{i}^{*}=\widetilde{r}, i \in I\right\}$, when $\beta<1$. Therefore, coexistence of conventions cannot emerge neither with heterogeneous neighborhood structures, nor with homogeneous ones where $r_{i}^{*}=r$, all $i$, but $r \neq 1, \widetilde{r}$. On the other hand, heterogeneous strategy configurations can sustain both homogeneous

\footnotetext{
${ }^{11}$ It suffices to repeat calculations performed in Appendix B to find necessary and sufficient conditions on $\beta$ such that an agent will always enlarge her radius irrespective of local frequencies.
} 
and heterogeneous networks. More importantly, the condition $\beta<1$ is necessary but not sufficient for the emergence of complete networks: when $\frac{1}{4} \leq \beta<1$ there might exist many SS where coexistence of conventions and heterogeneous neighborhood structures coexist. In addition, as happens when $\alpha=1$, the complete network (as well as any other homogeneous network with $r>1$ ) cannot be sustained in a SS by a strategy configuration characterized by coexistence of conventions (i.e. $\bar{c}<1)^{12}$.

Figs. $5 \mathrm{a}$ and $5 \mathrm{~b}$ plot, for the case $N=21$, average coordination and neighborhood radius as $(\beta, \theta)$ change. As expected, the long run behavior of the system is strongly affected by the level of unit cost. When $\beta<\frac{1}{4}$, full coordination together with complete networks always arise. However, when $\frac{1}{4} \leq \beta<1$, the mixed network externalities regime allows agents to trade-off between larger neighborhoods and higher coordination. Despite complete networks and full coordination are still very likely to emerge, the system may be dynamically trapped in partial coordination configurations where some agents hold small neighborhoods. This results contrasts with findings in Goyal \& Vega-Redondo (2001) and is due both to the assumption of indirect partner selection and to the constraints imposed by the underlying geography. Finally, if network externalities are 'always negative' $(\beta>1)$, average neighborhood sizes shrink very fast toward 1. As $\beta$ grows, the only stable neighborhood structure becomes indeed the minimal one (i.e. $\bar{r}_{m}=1$ ) for which we know there are many stable, heterogeneous, strategy configurations. Nevertheless, average coordination does not decrease dramatically: for all $N \geq 11, \gamma=2 \beta \leq 1.5$ and $\theta \in(0,1]$, $\bar{c}_{m}>0.90$ in $93.75 \%$ of all runs, as compared to $84.60 \%$ when $\alpha=0$, cf. Obs. 1.

The parameter $\alpha$ may be then interpreted as governing the network externality regime in the economy: for a given parameter setup $(N, \beta, \theta)$, the higher $\alpha$ the more network externalities induce agents to penalize larger neighborhood sizes. When one studies how average coordination and average neighborhood sizes vary when $\alpha$ smoothly changes in $[0,1]$, it is worth noting that, when $\beta$ is small, a system with $\alpha=1$ is able to reach coordination levels which are as high as the ones attained by any system with $\alpha<1$. However, as soon as $\alpha$ becomes less than 1, almost full coordination is reached because the complete network becomes stable and viable. On the contrary, systems with $\alpha=1$ attain high coordination together with very small neighborhood sizes and thus higher average net payoffs $^{13}$.

\footnotetext{
${ }^{12}$ The proof of this statement is analogous to that developed in Appendix A for the case $\alpha=1$. Notice also that when $\beta=1$ and full coordination applies, any neighborhood structure is stable because all payoffs are equal to zero.

${ }^{13}$ For instance, when $N=11, \beta=\frac{\gamma}{2}=10^{-6}$ and $\theta=1$, any system with $\alpha<1$ is able to reach full coordination in a complete network in almost $100 \%$ of runs. On the contrary, when $\alpha=1$ the analysis in the foregoing Sections shows that $\widehat{c} \simeq 0.945$ and $\widehat{R}=0.205$.
} 


\subsection{Higher-Dimensional Lattices, Neighborhood Specification and Coordination}

In the basic setup agents were placed in a 1-dimensional lattice with periodic boundaries (i.e. a circle). In this subsection we test whether (and in which ways) the behavior of the system is affected by this particular choice for the topology of interactions. In particular, we explore what happens to average coordination when: (a) agents are located in $v$-dimensional lattices, $v \geq 2$; and (b) we allow for notions of 'distance' between agents different from the Manhattan metrics $\left(d_{1}\right)$.

Let us start by assuming that each agent is associated to a node of a $v$-dimensional lattice with periodic boundaries. In this case, agent $i \in I$ has coordinates $\left(x_{i 1}, \ldots, x_{i v}\right)$. If the relevant metrics is still $d_{1}$, then the neighborhood of agent $i$ is defined as:

$$
V^{v}\left(r_{i t}\right)=:\left\{j \in I: 0<\sum_{h=1}^{v}\left|x_{i h}-x_{j h}\right| \leq r_{i t}\right\} \text {, }
$$

where $r_{i t} \in\{1,2, \ldots, \widetilde{r}\}$ and $\widetilde{r}=\left\lfloor N^{\frac{1}{v}}\right\rfloor$. It is easy to show that the size of a generic $r$-neighborhood in $v$ dimensions reads:

$$
\left|V^{v}(r)\right|=\sum_{m=0}^{\min \{r, v\}} \frac{(v+r-m) !}{m !(r-m) !(v-m) !} .
$$

Hence, for a given $v,\left|V^{v}(r)\right|$ grows as $r^{v}$ (and symmetrically, for a given $r$, as $v^{r}$ ).

Suppose that payoffs are still defined as in Eq. (3) and that $\alpha=1$ (i.e. we are in the standard case where the regime of network externalities allows for 'locally' positive but eventually 'negative' spillovers). Table 1 reports the results of some MC exercises for 2 and 3 -dimensional lattices, when $(\theta, \beta)$ belong to benchmark parametrizations. Since $\widetilde{r}$ depends on both $N$ and $v$, we study how average coordination and average neighborhood radius change when $v=1,2,3$ and either $\widetilde{r}$ or $N$ are kept fixed.

As Table 1 shows, in both setups with $v=2$ and $v=3$, average coordination is increasing with $\beta$ and $\theta$, while average radius is decreasing in $\beta$. Thus, the relationship between unit cost and frequency of neighborhood updating, on the one hand, and coordination and neighborhood size, on the other, is robust to changes in $v$. However, higher-dimensional lattices generally imply lower coordination. Furthermore, average neighborhood radius does not seem to be very much affected by $v$. The same pattern arises no matter one keeps constant the maximum attainable radius $\widetilde{r}$ (so that $N$ increases with $v$ ) or the population size $N$ (so that $\widetilde{r}$ decreases with $v$ ). Finally, as exemplified by the 2 -dimensional case, a larger population size implies lower coordination as soon as $v>1$.

This evidence is not surprising if one notices that, as the dimension of the lattice 
increases: (i) negative network externalities are more likely for a given $\beta$ (because gross payoffs are still bounded from above by 1, while neighborhood costs grow as $r^{v}$ ); (ii) the number of strategy configurations which are locally sustainable when all agents hold a radius $r=1$ increases (cf. Blume (1993)). Therefore, agents prefer to hold a radius $r=1$ even when $v>1$ because of stronger NNE. At that point, the system is more likely to be trapped in a stable strategy configuration characterized by lower coordination.

Furthermore, the number of neighbors that two adjacent agents have in common decreases with $v$ (if the size of the neighborhood remains constant). Hence, as the population size increases, the degree of anonymity in the lattice increases (cf. Berninghaus \& Schwalbe (1996)). In turn, this implies that the constraints imposed by the geography of interactions on stable strategy configuration are no longer stringent as in the 1-dimensional case (cf. App. C). Thus, low coordination configurations are still reachable even when $N$ grows.

Finally, when $v \geq 2$ the metrics with which we endow the space where agents are located does affect long-run patterns of coordination. Suppose for example that the distance between two agents $i$ and $j$ is defined as:

$$
d_{i j}=\max _{h=1, \ldots, v}\left\{\left|x_{i h}-x_{j h}\right|\right\}
$$

A neighborhood with radius $r$ will then have a hypercubic shape (i.e. a Moore's neighborhood) instead of a hyperspheric shape (i.e. a Von Neumann's neighborhood) ${ }^{14}$. This new geography of interactions usually entails higher coordination levels, as compared to the standard case where neighborhoods are defined employing the Manhattan metrics. Indeed, when $N$ is kept fixed as $v=2,3$, the society achieves almost full coordination in all parametrizations (i.e. $\bar{c}_{m}>0.99$ in $95 \%$ of all runs).

\subsection{Pareto-Efficient vs. Risk-Dominant Nash Equilibria}

The simple model presented in Section 3 may be easily employed to address the issue of convergence to Pareto-efficient (PE) vs. risk-dominant (RD) equilibria. Suppose indeed that agents play generic coordination games (instead of pure coordination ones). In this case, if agent $i$ plays $s_{i} \in\{-1,1\}$ when agent $j^{\prime} s$ strategy is $s_{j} \in\{-1,1\}$, her stage-game

\footnotetext{
${ }^{14}$ The size of a $r$-radius Moore's neighborhood in $v$ dimensions is thus $(2 r+1)^{v}-1$, which of course is larger than the size of a Von Neumann's neighborhood. Moreover, two adjacent agents holding $r$ Moore's neighborhoods have more neighbors in common than two adjacent agents with $r$-Von Neumann's neighborhoods.
} 
payoff is given by:

\begin{tabular}{c|c|c|}
$G\left(s_{i}, s_{j}\right)$ & $s_{j}=+1$ & $s_{j}=-1$ \\
\hline$s_{i}=+1$ & $a$ & $b$ \\
\hline$s_{i}=-1$ & $c$ & $d$ \\
\hline
\end{tabular}

where $a>c, b<d$ and $a>d$ (i.e. the Nash equilibrium $(+1,+1)$ is Pareto efficient). Recent studies have pointed out that Pareto efficiency might not generally be the relevant selection principle whenever the other Nash equilibrium (i.e. $(-1,-1))$ is risk-dominant, i.e. if $c+d>a+b$. More specifically, Kandori et al. (1993), Ellison (1993), Young (1996) and Blume (1993) have shown that, whenever agents repeatedly interact (either locally or globally) with the same set of partners and employ best-reply rules with trembles, the unique long-run outcome is the establishment of the risk-dominant convention (all play -1 ). Convergence to the PE convention (all play +1 ) is however restored when players are spatially located but they can indirectly select their partners by moving across locations (cf. Ely (1996), Oechssler (1997) and Dieckemann (1999)). In general, Pareto efficiency is typically the case whenever agents have some flexibility in selecting their partners and they are able to 'isolate' themselves within islands of coordination (see e.g. Goyal \& Vega-Redondo (2001, p. 8)). However, when interaction structures are made endogenous, efficiency results strongly depend on the details of network evolution. For instance, when unit link costs are small enough, Goyal \& Vega-Redondo (2001) find that the system almost surely converges to the RD outcome, while in Jackson \& Watts (2000) the configuration where all agents play -1 is not the unique (evolutionary) stable state.

To explore efficiency issues in the present model, we considered the basic setup where $v=1$ and $\alpha=1$ and we let for the sake of simplicity $a=2, b=0, d=1$ and $c \in[0,2)$. Notice that if $c \in[0,1)$ the PE Nash equilibrium is also RD, while if $c \in(1,2)$ the NE $(-1,-1)$ is RD. Finally, if $c=1$ the two pure-strategy equilibria are risk-equivalent. Table 2 shows for $N=51$ and some benchmark parametrization for $(\beta, \theta)$ MC means of the average strategy $\left(\bar{s}_{m}\right)$ and average radius $\left(\bar{r}_{m}\right)$, as $c=0.00,1.00,1.99$.

Across all parametrizations, the system displays a clear tendency toward the riskdominant equilibrium. In contrast with existing results, the regime of network externalities studied here restores convergence to the RD outcome even when unit costs are large enough. In fact, when the $\mathrm{RD}$ equilibrium is unique (i.e. $c=0$ and $c=1.99$ ), the system converges to homogeneous configurations when all agents play the RD strategy (and also $\mathrm{PE}$ only when $c=0$ ). Notice however that when both equilibria are risk-equivalent, $50 \%$ of agents play the PE equilibrium and this frequency increases when unit costs are small and the frequency of neighborhood updating is large. In these cases, players enjoy larger neighborhoods and are able to better coordinate on the PE equilibrium. 


\section{Conclusions}

The details of the process governing the coevolution of expectations formation, individual choices and interaction structure might strongly affect the long-run outcome of a coordination game repeatedly played in large populations.

To investigate this issue, we have presented a model in which myopic, spatially located individuals play a pure coordination game with their nearest neighbors. Key features of the models are, first, that agents are allowed, in each period, to adjust both current stagegame strategies and neighborhood size with an exogenously given probability. Since this probability can be treated as a system parameter, one can address the question whether a higher frequency of endogenous neighborhood formation entails higher aggregate coordination. Second, neighborhood formation is itself a local interaction process, because any agent can only enlarge or shrink by one unit her interaction radius. Finally, we analyze a system characterized by local network externalities which might be positive when the neighborhood size is relatively small, but they eventually become negative when the latter becomes too large.

We show that the system almost always converges to a set of absorbing states in which full coordination, as well as coexistence of conventions, are both possible. However, if full coordination arises, all players must hold the smallest as possible neighborhood. On the contrary, long-run coexistence of conventions can arise together with many, possibly heterogeneous, neighborhood structures. Nevertheless, computer simulations show that the system is able to reach very large average coordination levels, together with small average neighborhood sizes, for almost all parametrizations. We also find that the rate of neighborhood updating negatively affects average coordination levels. This raises the question of how coordination levels with endogenous neighborhoods compare to the case in which interaction structures are static. Simulations indicate that, for a large class of exogenously given interaction structures and system parameters, a population with a small but positive rate of endogenous neighborhood formation always reaches higher coordination. In addition, we find that large societies perform better than small ones provided that individuals initially hold, on average, large neighborhoods.

Finally, we explore the behavior of the model when one gradually departs from the basic setup by assuming that: (i) network externalities might be positive for all neighborhood sizes; (ii) agents are located in lattices with dimension higher than one; and (iii) agents play generic coordination stage-games where a trade-off arises between Pareto-efficient and risk-dominant Nash equilibria.

More generally, the robustness of the foregoing results should be checked with respect to a number of key assumptions. First, one might study the effects of introducing individual decision rules different from deterministic best-reply (e.g. best-reply with mistakes, log- 
linear decision rules, etc.). Along the same lines, the consequences of assuming alternative tie-breaking rules and updating schemes should be thoroughly analyzed. For example, one could experiment with agents who always randomize when ties occur. In addition, one might explore the dynamic properties of the system in presence of a synchronous - or an incentive-based - updating scheme governing how many agents (and who) are allowed to update their state in each period (cf. Page (1997)). Second, introducing non-linear neighborhood cost functions, as well as gross payoffs from coordination which themselves depend on the distance between agents, might allow a deeper understanding of the trade-off between negative and positive network externalities.

\section{References}

Akerlof, G. (1997), 'Social distance and social decisions', Econometrica 65, 1005-1027.

Arnott, R. \& Small, K. (1994), 'The economics of traffic congestion', American Scientist 82, 446-455.

Bala, V. \& Goyal, S. (2000), 'A non-cooperative model of network formation', Econometrica 68(5), 1181-1229.

Berninghaus, S. \& Schwalbe, U. (1996), 'Conventions, local interaction and automata networks', Journal of Evolutionary Economics 6, 297-312.

Blume, L. (1993), 'The statistical mechanics of strategic interaction', Games and Economic Behaviour 5, 387-424.

Blume, L. (1995), 'The statistical mechanics of best-response strategy revision', Games and Economic Behavior 11, 111-145.

Cowan, R., Cowan, W. \& Swann, P. (1998), Waves in consumption with interdependence among consumers. mimeo, University of Western Ontario, Canada.

Dieckemann, T. (1999), 'The evolution of conventions with mobile players', Journal of Economic Behavior and Organization 38, 93-111.

Droste, E., Gilles, R. \& Johnson, C. (2000), Evolution of conventions in endogenous social networks. Unpublished Manuscript, CentER, Tilburg University, The Netherlands.

Economides, N. (1996), 'The economics of networks', International Journal of Industrial Organization 14(2).

Ellison, G. (1993), 'Learning, local interaction and coordination', Econometrica 61(5), 1047-1071.

Ely, J. (1996), Local conventions. Unpublished Manuscript, Department of Economics, Northwestern University. 
Goyal, S. \& Janssen, M. (1997), ‘Non-exclusive conventions and social coordination', Journal of Economic Theory 77, 34-57.

Goyal, S. \& Vega-Redondo, F. (2001), Learning, network formation and coordination. Unpublished Manuscript, Erasmus University, Rotterdam.

Gupta, A., Stahl, D. \& Whinston, A. (1995), The internet: A future tradegy of the commons ? Unpublished Manuscript, The University of Texas at Austin.

Jackson, M. \& Watts, A. (1999), The evolution of social and economic networks. Unpublished Manuscript, Division of Humanities and Social Sciences, Caltech, Pasadena, CA and Vanderbilt University, Nashville, TN.

Jackson, M. \& Watts, A. (2000), On the formation of interaction networks in social coordination games. Unpublished Manuscript, Division of Humanities and Social Sciences, Caltech, Pasadena, CA and Vanderbilt University, Nashville, TN.

Kandori, M., Mailath, G. \& Rob, R. (1993), 'Learning, mutation and long run equilibria in games', Econometrica 61(1), 29-56.

Kirman, A. (1997), 'The economy as an evolving network', Journal of Evolutionary Economics 7, 339-353.

Mailath, G., Samuelson, L. \& Shaked, A. (2000), Evolution and endogenous interactions, in U. Pagano \& A. Nicita, eds, 'The Evolution of Economic Diversity', Siena, Routledge.

Miller, D. (1963), The study of social relationships: Situations, identity and social interactions, in S. Kock, ed., 'Psichology: A Study of a Science, Vol. 5', New York, McGraw Hill.

Mitchell, C. (2000), Discussing informational technologies and internet in terms of the positive and negative externalities they create. Unpublished Manuscript, American Political Science Meeting, Washington D.C.

Oechssler, J. (1997), 'Decentralization and the coordination problem', Journal of Economic Behavior and Organization 32, 119-135.

Page, S. (1997), 'On incentives and updating in agent based models', Computational Economics 10, 67-87.

Skyrms, B. \& Pemantle, R. (2000), 'A dynamic model of social network formation', Proceedings of the National Academy of Sciences 97, 9340-9346.

Watts, D. (2001), 'A dynamic model of network formation', Games and Economic Behavior 34, 331-341.

Young, H. (1996), 'The economics of convention', Journal of Economic Perspectives 10(2), 105-122.

Young, H. (1998), Individual Strategy and Social Structure, Princeton, Princeton University Press. 


\section{Appendices}

\section{A Proofs}

\section{Proof of Prop. 2}

Consider (wlog) individual updating rules when $\theta=1$. Given the current strategy profile, the agent drawn to update her current state $(s, r)$ faces the following relevant payoff matrix:

\begin{tabular}{|c|c|c|c|}
\hline & $r-1$ & $r$ & $r+1$ \\
\hline$s=+1$ & $x_{i, r-1}-\gamma(r-1)$ & $x_{i, r}-\gamma r$ & $x_{i, r+1}-\gamma(r+1)$ \\
\hline$s=-1$ & $1-x_{i, r-1}-\gamma(r-1)$ & $1-x_{i, r}-\gamma r$ & $1-x_{i, r+1}-\gamma(r+1)$ \\
\hline
\end{tabular}

If given local frequency profile $\left(x_{i, r-1}, x_{i, r}, x_{i, r+1}\right)$ no ties occur, then best-replies are unique. If, on the contrary, some ties occur, redefine the payoff matrix by adding some $\varepsilon>0$ to the entry associated to the choice preferred under the relevant tie-breaking rule (see Section (3)). Once again, the resulting embedded game is such that, for any given strategy configuration, there is a unique best-response $\left(s^{*}, r^{*}\right)$ for any individual facing updating. Therefore, for any given $N \geq 5, \theta \in(0,1], \gamma>0$ and $\Omega_{0} \in \Gamma(N)$, the Markov chain governing the evolution of the process $\left\{\Omega_{t}, t \geq 1\right\}$ has the so-called 'unique best-reply property' (see Blume (1995)). For such Markov processes, we know that there is a one-to-one correspondence between absorbing states and ergodic sets. Hence, finite-time convergence to an absorbing state is a probability one event in our system. Finally, notice that the tiebreaking assumption concerning the case when $\left(s, r_{i, t}+1\right)$ and $\left(s, r_{i, t}-1\right)$ are the unique solutions of (6) for $s \in\{-1,1\}$ (i.e. always choose $\left(s, r_{i, t}-1\right)$ ), is not very restrictive. Indeed, let $h=0,1, \ldots, 2(r-1)$ the number of agents playing +1 in $V_{r-1}$ and $k_{r}=0,1,2$ the number of agents playing +1 in the set $V_{i, r} \backslash V_{i, r-1}$. It is easy to show that a tie occurs (when $s=+1$ ), i.e.:

$$
x_{r-1}-\gamma(r-1)=x_{r+1}-\gamma(r+1)>x_{r}-\gamma r
$$

iff $h \geq r-k_{r}$ and:

$$
\gamma=\frac{(r-1)\left(k_{r}+k_{r+1}\right)-2 h}{4(r+1)(r-1)}>\frac{(r-1) k_{r}-h}{2 r(r-1)} .
$$

Numerical experiments show that the set of all $\gamma$ satisfying these conditions has cardinality that grows as $N^{2}$. Hence, if $\gamma$ lies in a dense subset of $R_{+}$, the considered tie-breaking rule is not relevant and the embedded game has the unique best-reply property anyway.

\section{Proof of Prop. 3}

Proof of Point 1. We need to show that: (i) the configuration $\left\{\left(s_{i}, r_{i}\right)=(+1,1)\right.$, all $i \in I\}$ is a SS; and that (ii) given that all agents play $s_{i}=+1$ only $r_{i}=1$ all $i$ can be a 
SS. Notice that payoff symmetry with respect to strategies allows us to avoid treating the case $s_{i}=-1$, all $i$. Moreover, as SS conditions for strategy updating are contained in SS conditions for neighborhood updating, it suffices to check the latter. When all $s_{i}=+1$, then $x_{i}(r)=1 \forall r=1, \ldots, \widetilde{r}$. Hence, for $r=2, \ldots, \widetilde{r}-1$, generic payoff matrices read:

\begin{tabular}{|c|c|c|c|}
\hline & $r-1$ & $r$ & $r+1$ \\
\hline$s=+1$ & $1-\gamma(r-1)$ & $1-\gamma r$ & $1-\gamma(r+1)$ \\
\hline$s=-1$ & $-\gamma(r-1)$ & $-\gamma r$ & $-\gamma(r+1)$ \\
\hline
\end{tabular}

As $1-\gamma r \geq-\gamma r$ for any $r$ the strategy configuration $s_{i}=+1$, all $i$, is stable for any $r$. However, as payoffs are decreasing in $r$, any agent will shrink until $r_{i}=1$, all $i$.

Proof of Point 2. Consider wlog $N>3$ odd. Let us start by observing that there are no SS action configurations where exactly 1 or $N-1$ agents choose +1 (or symmetrically -1 ). Indeed, suppose $s_{1}=-1, s_{i}=+1$ for $i=2, \ldots, N$. Then $x_{1}(r)=1$ for any $r$. Hence, even if all remaining strategies and radii are stable (e.g. because all $r_{i}=1, i \geq 2$ ), agent 1 will always switch. As a result, if strategy heterogeneity is the case, then $\widehat{s} \neq\left\{-\frac{N-1}{N},+\frac{N-1}{N}\right\}$. To show point 2., we need to prove that, for some neighborhood configurations, there exist a SS strategy configuration s.t. $\widehat{s} \in\left\{-\frac{N-2}{N},+\frac{N-2}{N}\right\}$ for any $N$ and $\gamma$. Consider for instance $r_{i}^{*}=1$, all $i$. Then any SS strategy configuration such that $\widehat{s} \in\left\{-\frac{N-2}{N},+\frac{N-2}{N}\right\}$ is a SS. To see why, consider agents playing +1 (a symmetric argument holds for agents playing -1 ). SS conditions can be rewritten as follows: $\gamma \geq \max \left\{1-x_{1}-x_{2}, x_{2}-x_{1}\right\}$. As far as local frequencies $x_{1}$ and $x_{2}$ are concerned, there can be at most three types of agents, namely: (i) $\left(x_{1}, x_{2}\right)=(1,1)$; (ii) $\left(x_{1}, x_{2}\right)=\left(1, \frac{3}{4}\right)$;(iii) $\left(x_{1}, x_{2}\right)=\left(\frac{1}{2}, \frac{1}{2}\right)$. In all such cases, the SS condition above reads $\gamma \geq 0$, which is always true.

Proof of Point 3. We need to show that for any $\left\{s_{i}, i \in I\right\}$ s.t. $|\widehat{s}| \neq 1$,homogenous radius configurations are only possible if all agents choose $r_{i}=r=1$. First, notice that $r_{i}=\widetilde{r}$, all $i$ cannot be a SS. If it were, then it must be that for all $i, x_{i}(\widetilde{r}) \geq \frac{1}{2}$ if $s_{i}^{*}=+1$, while $x_{i}(\widetilde{r}) \leq \frac{1}{2}$ if $s_{i}^{*}=-1$. Letting $x^{*}$ be the global frequency of agents playing +1 , then $x_{i}(\widetilde{r})=\frac{N x^{*}-1}{N-1}$ if $s_{i}^{*}=+1$ and $x_{i}(\widetilde{r})=\frac{N x^{*}}{N-1}$ if $s_{i}^{*}=-1$. Hence, in a SS: $x^{*} \geq \frac{N+1}{2 N}$ if $s_{i}^{*}=+1$ while $x^{*} \leq \frac{N-1}{2 N}$ if $s_{i}^{*}=-1$. By heterogeneity, the two inequalities should be satisfied simultaneously, which gives a contradiction.

Second, any configuration in which $r_{i}=2, \ldots, \widetilde{r}-1$, all $i$, cannot be a SS either. If it were, SS conditions would jointly imply (cf. Lemma 1) that $x_{i}^{*}(r)>\frac{1}{2}$ if $s_{i}^{*}=+1$ and $x_{i}^{*}(r)<\frac{1}{2}$ if $s_{i}^{*}=-1$. However, if a heterogeneous configuration is to be stable, any two adjacent agents, say $i$ and $i+1$, such that $s_{i}^{*}=+1$ and $s_{i+1}^{*}=-1$ must be locally stable. As both holds a $r$-radius neighborhood, then $V_{i, r}=\{i-r, i-r+1, \ldots, i-1, i+1, i+2, \ldots, i+r\}$ and $V_{i+1, r}=\{i-r+1, \ldots, i-1, i, i+2, \ldots, i+r, i+r+1\}$. Hence $V_{i, r} \cap V_{i+1, r}=\{i-r+1, \ldots$, $i-1, i+2, \ldots, i+r\}$. Let us call $x_{c}$ the frequency of agents playing +1 in $V_{i, r} \cap V_{i+1, r}$ whose size is $2(r-1)$. Then, since $s_{i}^{*}=+1$ and $s_{i+1}^{*}=-1$ :

$$
x_{i}=\frac{2(r-1) x_{c}+1_{\left(s_{i-r}=+1\right)}}{2 r} \text { and } x_{i+1}=\frac{2(r-1) x_{c}+1+1_{\left(s_{i+r+1}=+1\right)}}{2 r},
$$


where $1_{\left(s_{j}=+1\right)}=1$ if $s_{j}=+1$ and 0 otherwise. By the inequality above, we must have that:

$$
\left\{\begin{array}{c}
2(r-1) x_{c}+1_{\left(s_{i-r}=+1\right)}>r \\
2(r-1) x_{c}+1+1_{\left(s_{i+r+1}=+1\right)}<r
\end{array}\right.
$$

or $r-1_{\left(s_{i-r}=+1\right)}<2(r-1) x_{c}<r-1-1_{\left(s_{i+r+1}=+1\right)}$, which is impossible as $\max \left\{1_{\left(s_{i-r}=+1\right)}-\right.$ $\left.1_{\left(s_{i+r+1}=+1\right)}\right\}=1$.

Proof of Point 4. To show point 4, we will provide examples supporting the statement that, depending on $(\gamma, N)$, there exist SS sustaining heterogeneous neighborhood sizes with some agents choosing $r_{i}^{*}=1$ and some other agents choosing $r_{i}^{*} \in\{2, \ldots, \widetilde{r}-1, \widetilde{r}\}$. To show that a given strategy-radius configuration is a SS, one should check if, for any agent, SS inequalities are satisfied. In the following Lemma, we will present an easier way to check for local SS conditions. Indeed, for an individual state $\left(s_{i}, r_{i}\right)$ to be stable, only the number of agents playing +1 in $V_{i}(r-1)$, as well as the 4 agents playing +1 in $V_{i}(r) \cap V_{i}(r-1)$ and in $V_{i}(r+1) \cap V_{i}(r)$, matter.

Lemma 5 (Characterization of Local SS Conditions) Assume (wlog) that $s_{i}=+1$ (symmetric results hold for $s=-1$ ). Let $V_{r} / V_{r-1}=\left\{j \in I: j \in V_{r}, j \notin V_{r-1}\right\}$. Then:

1. Suppose that $r_{i}=1$. Let $h=0,1,2$ the number of agents $i$ playing +1 in $V_{2}$ and $k=0,1,2$ the number of agents $i$ playing +1 in $V_{2} / V_{1}$. Then $r=1$ is locally stable for agent $i$ if and only if $h=1,2$ (i.e. $h \neq 1), k \in\{0,1,2\}$ and $\gamma$ satisfy the following conditions:

\begin{tabular}{|c|c|c|c|c|c|c|c|c|c|c|c|c|c|c|c|}
\hline \multirow{2}{*}{$h=1$} & \multicolumn{5}{|c|}{$k=0$} & \multicolumn{5}{|c|}{$k=1$} & \multicolumn{5}{|c|}{$k=2$} \\
\hline & - & - & $\begin{array}{c}\gamma \geq \frac{1}{4} \\
(+)\end{array}$ & + & - & - & - & $\begin{array}{c}\forall \gamma \\
(+)\end{array}$ & & + & + & & $\begin{array}{c}\gamma \geq \frac{1}{4} \\
(+)\end{array}$ & + & + \\
\hline$h=2$ & - & + & $\begin{array}{l}\forall \gamma \\
(+)\end{array}$ & + & - & - & + & $\begin{array}{c}\quad \forall \gamma \\
(+)\end{array}$ & + & + & + & + & $\begin{array}{c}\forall \gamma \\
(+)\end{array}$ & & + \\
\hline
\end{tabular}

Illustrations of local strategy configurations consistent with correspondent values of $(h, k)$ are also shown. Notice that also configurations obtained from the ones reported by simply switching neighbors symmetrically are $S S$.

2. Suppose that $r_{i}=\widetilde{r}$. Let $h=0,1, \ldots, 2(\widetilde{r}-1)$ the number of agents $i$ playing +1 in $V_{\widetilde{r}-1}$ and $k=0,1,2$ the number of agents $i$ playing +1 in $V_{\widetilde{r}} / V_{\widetilde{r}-1}$. Then $r=\widetilde{r}$ is locally stable for agent $i$ if and only if (supposing wlog $N$ odd): $k=2, \frac{N-3}{2}=r-1 \leq h \leq$ $2 \widetilde{r}-3=N-4$ and

$$
\gamma \leq \frac{2(\widetilde{r}-1)-h}{2 \widetilde{r}(\widetilde{r}-1)}=\frac{2(N-3-h)}{(N-1)(N-3)}
$$

3. Suppose that $r_{i}=2, \ldots, \widetilde{r}-1$. Let $h$ the number of agents playing +1 in $V_{r-1}$ and $k_{r}=0,1,2$ be the number of agents playing +1 in the set $V_{i, r} \backslash V_{i, r-1}$. Then $r=r_{i}$ is 
locally stable for agent $i$ if and only if $k_{r} \neq\{0,1\}$ and:

\begin{tabular}{|c|c|c|}
\hline & $k_{r+1}=0,1$ & $k_{r+1}=2$ \\
\hline$k_{r}=2$ & $h=r-1, r-2, \ldots, 2 r-3$ & $h=\frac{r-1}{r-2}, r-\ldots, 2 r-3$ \\
& $\gamma \leq \frac{2(r-1)-h}{2 r(r-1)}$ & $\frac{2(r-1)-h}{2 r(r+1)} \leq \gamma \leq \frac{2(r-1)-h}{2 r(r-1)}$ \\
\hline
\end{tabular}

Proof. We prove here only the case $r_{i}=1$ (proofs of cases 2 and 3 are similar but require heavier computations). Consider first SS inequalities that $(h, k)$ must satisfy in order for $(+1,1)$ to be stable for given the strategy choice $s_{i}=+1$. Since $x_{1} \geq \max \left\{\frac{1}{2}, x_{2}-\gamma\right\}$, then $\frac{h}{2} \geq \max \left\{\frac{1}{2}, \frac{h+k}{4}-\gamma\right\}$. This implies $h>1$ and $k-h \leq 4 \gamma$, which is always satisfied for any $\gamma>0$ unless when $h=1$ and $k=2$, where we need $\gamma \geq \frac{1}{2}$. If we now add constraints implied by the inequalities concerning a strategy switch, then the only additional restrictions come from the fact that $x_{2} \geq 1-x_{1}-\gamma$, which implies $4-3 h-k \leq 4 \gamma$. As $h=1,2$ and $k=0,1,2$, the (lhs) of the last inequality is always $\leq 0$ unless $(h, k)=(1,0)$, where we need $\gamma \geq \frac{1}{4}$.

Given Lemma 5, it is now easy to see that the following configurations are SS in which strategy heterogeneity sustains neighborhood homogeneity.

Example 6 Consider the following example $(N=21)$. Then, the following configuration: $\{-1,-1,-1,+1,+1,+1,+1,+4,+4,-10,+2,+1,+1,-4,-1,-1,-1,-1,-1,-1,-1\}$, where (sign) $x$ means $s=($ sign $) 1$ and $r=x$, is a $S S$ for $\gamma \leq 8 /(7 * 21)$.

To see that this is the case, it suffices to apply conditions given in Lemma 5 to any agent $i$.

\section{B The Effect of Negative Local Network Externalities}

In this Section we will better characterize the effect of negative local externalities. We will focus on the extreme case in which an agent currently playing $(+1, r)$, no matter local frequencies $\left(x_{r-1}, x_{r}, x_{r+1}\right)$, would have an incentive to either always shrink her radius to $r-1$, keep $r$, or enlarge to $r-1$. We will show that negative network externalities engenders an asymmetric effect on players incentives because there exist regions of the parameter space in which agents are always willing to shrink no matter local frequencies, but the same is not true as far as incentives to keep or enlarge are concerned. More formally:

Proposition 7 Consider an agent playing (wlog) +1 and holding a r-radius neighborhood, $r \geq 2$. Then:

1. If $\gamma>\gamma^{*}(r)=\frac{1}{2(r-1)}$ the agent will switch to $(r-1,-1)$ no matter local frequencies.

2. There are no $\gamma$ s.t. given $N$ an agent will switch to $r+1$ or keep $r$ no matter local frequencies. 
Proof. Consider Point 1. The agent will shrink iff $x_{r}-x_{r-1}<\gamma$ and $x_{r+1}-x_{r-1}<2 \gamma$. If we let $x_{r-1}=h / 2(r-1), x_{r}=\left(h+k_{r}\right) / 2 r$ and $x_{r+1}=\left(h+k_{r}+k_{r+1}\right) / 2(r+1)$, then the latter inequalities are satisfied iff:

$$
\frac{r k_{r}-h-k_{r}}{2 r(r-1)}<\gamma \quad \text { and } \quad \frac{r k_{r}+(r-1) k_{r+1}-h-\left(h+k_{r}\right)}{4(r+1)(r-1)}<\gamma
$$

The extreme case for an agent willing to shrink is $k_{r+1}=2, k_{r}=2$ and $h+k_{r}=r$ (i.e. $h=r-2$ ). In this circumstance (and consequently in any other one), the agent will shrink iff $\gamma>\gamma^{*}=[2(r-1)]^{-1}$. When allowed to update her strategy (e.t. else being equal), her local frequency will be $x=x_{r-1}=(r-2)[2(r-1)]^{-1}<\frac{1}{2}$, so that the agent will switch to -1 .

Consider now point 2. For an agent to keep $r$, it must be that:

$$
\frac{(r-1) k_{r}-h}{2 r(r-1)} \geq \gamma \text { and } \frac{r k_{r+1}-\left(h+k_{r}\right)}{2 r(r+1)} \leq \gamma .
$$

Here the extreme case is: $k_{r+1}=2, k_{r}=0$ and $h+k_{r}=h=2(r-1)$, so that $x_{r}=$ $(r-1) / r \geq 1 / 2$. The first inequality reads $-(1 / r) \geq \gamma$ which is not possible. Therefore, the agent will never keep $r$ irrespective of frequencies. Finally, suppose that an agent would always be willing to enlarge. Then it must be that:

$$
\frac{(r-1) k_{r}+(r-1) k_{r+1}-2 h}{4(r+1)(r-1)}>\gamma \text { and } \frac{r k_{r+1}-\left(h+k_{r}\right)}{2 r(r+1)}>\gamma .
$$

In the extreme case (i.e. $k_{r+1}=0, k_{r}=2$ and $h+k_{r}=2 r$ ) the inequalities above cannot be simultaneously satisfied.

Finally notice that, for any given $\gamma$, there exists a threshold $r^{*}(\gamma)=(1+2 \gamma) / 2 \gamma$ s.t. if an agent holds a radius $r>r^{*}(\gamma)$ then it will always shrink and switch action. However, such $r^{\prime}$ s are possible only if $N \geq N(\gamma)=\left\lfloor 2 r^{*}(\gamma)+1\right\rfloor=\left\lfloor\frac{1+3 \gamma}{\gamma}\right\rfloor+1$.

\section{Coexistence of Conventions and Population Size}

We provide here the intuition behind the increase in average coordination due to a growing population size (when the interaction radii are drawn at random from $\{1,2, \ldots,\lfloor N / 2\rfloor\}$ ). Suppose that $\theta=0$ (the conclusion will hold a fortiori when $\theta>0$ ). Then:

Proposition 8 For any given $N$, let a heterogeneous strategy configuration be given. For any pair of adjacent agents $(i, i+1)$ playing opposite strategies, say $(+1,-1)$ and holding radii $\left(r_{i}, r_{i+1}\right)=(r+p, r)$, where $r \geq 1$ and $p \geq 0$, then the number of common neighbors playing +1 who are necessary for the above configuration to be locally stable increases as $r$ increases.

Proof. Suppose first that $p>0$. If $\left(r_{i}, r_{i+1}\right)=(r+p, r)$ then $V_{i}=\{i-r-p, \ldots$, $i-r, i-r+1, \ldots, i-1, i+1, i+2, \ldots, i+r+1, i+r+2, \ldots, i+r+p\}$ and $V_{i+1}=\{i+1-r, \ldots, i$ $, i+2, \ldots, i+r+1\}$. Hence $V_{i} \cap V_{i+1}=\{i-r+1, \ldots, i-1, i+2, \ldots, i+r+1\}$. There are $2 r-1$ common neighbors and $2 p$ agents belonging to $V_{i} \backslash V_{i+1}$. Let $h$ and $h_{p}$ the number 
of agents playing +1 respectively in $V_{i} \cap V_{i+1}$ and $V_{i} \backslash V_{i+1}$. Hence local frequencies must satisfy:

$$
x_{i}(r+p)=\frac{h_{p}+h}{2(r+p)} \geq \frac{1}{2}, \quad x_{i+1}(r)=\frac{h+1}{2 r} \leq \frac{1}{2},
$$

which is possible iff $h_{p} \geq p+1$ and $r+p-h_{p} \leq h \leq r-1$. As $r$ grows, $h$ must grow. If conversely $p=0$, it is easy to show along the same lines that we need $h=r-1$.

Therefore, if $N$ grows and $r_{i} \in\{1,2, \ldots,\lfloor N / 2\rfloor\}$, agents will hold on average larger neighborhoods. Consequently, the number of common neighbors choosing +1 must grow for any pair of adjacent agents playing opposite strategies. This will of course systematically destroy heterogeneous strategy configurations as $N$ becomes larger. 


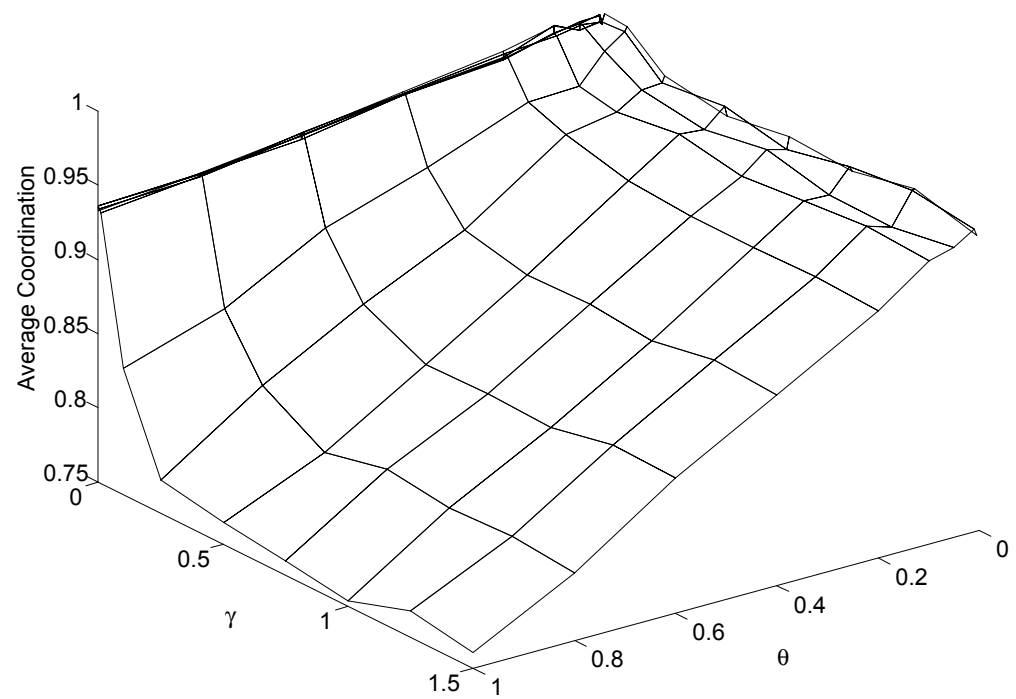

Figure 1(a). Means of MC average coordination distributions $\left\{\bar{c}_{m}, m=1, \ldots, M\right\}$ as a function of the unit neighborhood cost $\gamma$ and the frequency of neighborhood updating $\theta$. Population Size $N=21$. MC Sample Size $M=1000$.

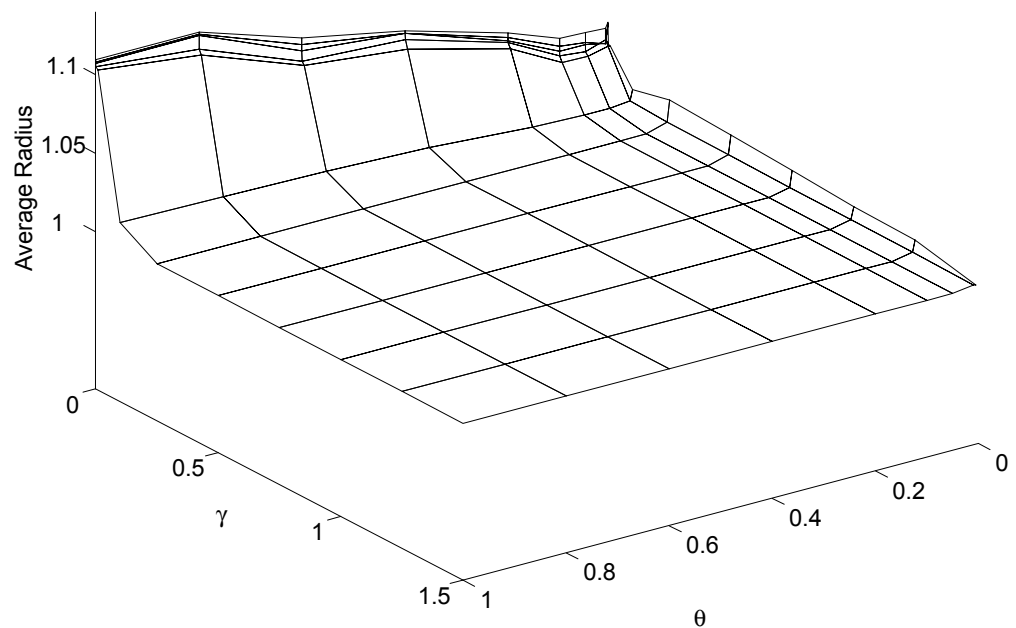

Figure 1(b). Means of MC average radius distributions $\left\{\bar{r}_{m}, m=1, \ldots, M\right\}$ as a function of the unit neighborhood cost $\gamma$ and the frequency of neighborhood updating $\theta$. Population Size $N=21$. MC Sample Size $M=1000$. 


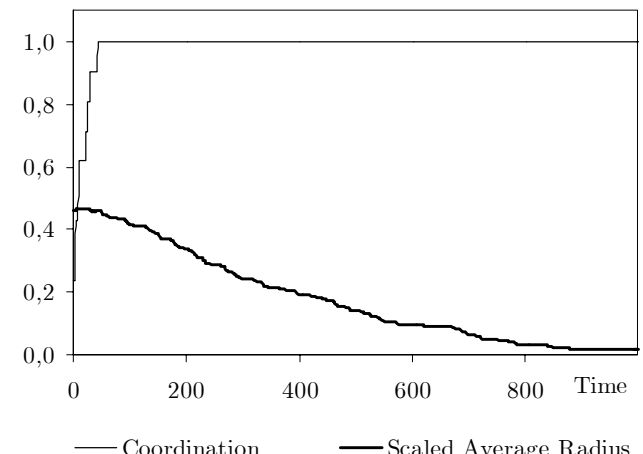

(a)

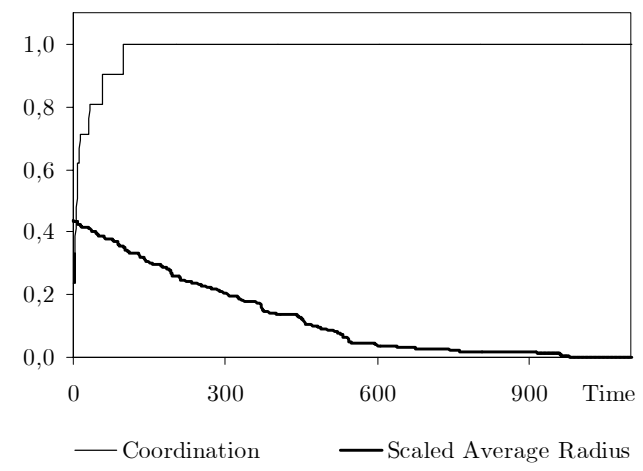

(c)

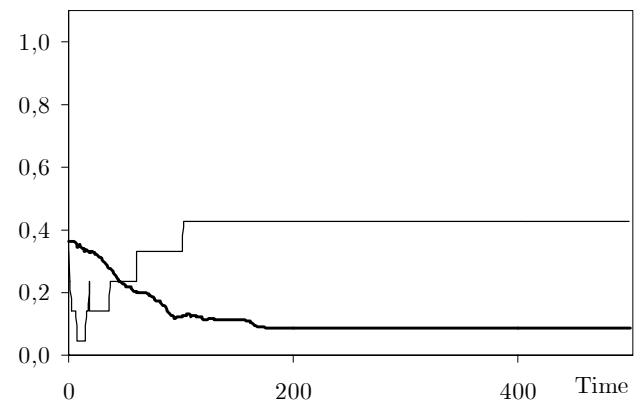

- Coordination $\quad$-Scaled Average Radius

(b)

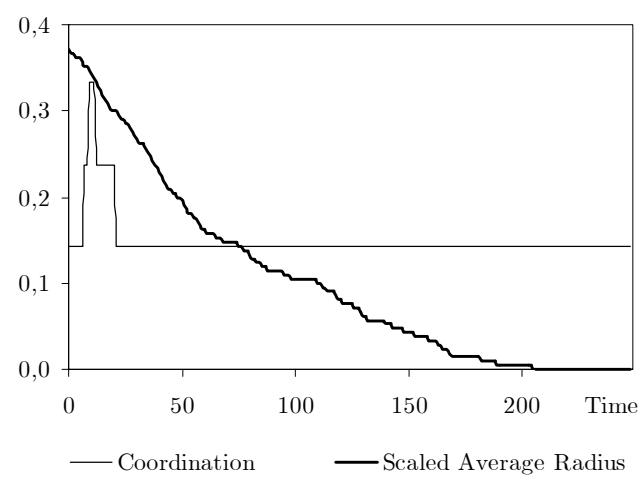

(d)

Fig. 2: Time-Evolution in a Typical Run of average coordination $\bar{c}$ and scaled average radius $\bar{r}$. Panels: (a) Small $\gamma$, small $\theta$; (b) Small $\gamma$, large $\theta$; (c) Large $\gamma$, small $\theta$; (d) Large $\gamma$, large $\theta$.

System Parameters: $\gamma \in\left\{10^{-6}, 1.5\right\}, \theta \in\{0.1,1.0\}, N=11$. Average Radius is scaled by $(N-1) / 2$ so as to match unit interval. Average Radius is scaled by $(N-1) / 2$ so as to match unit interval.

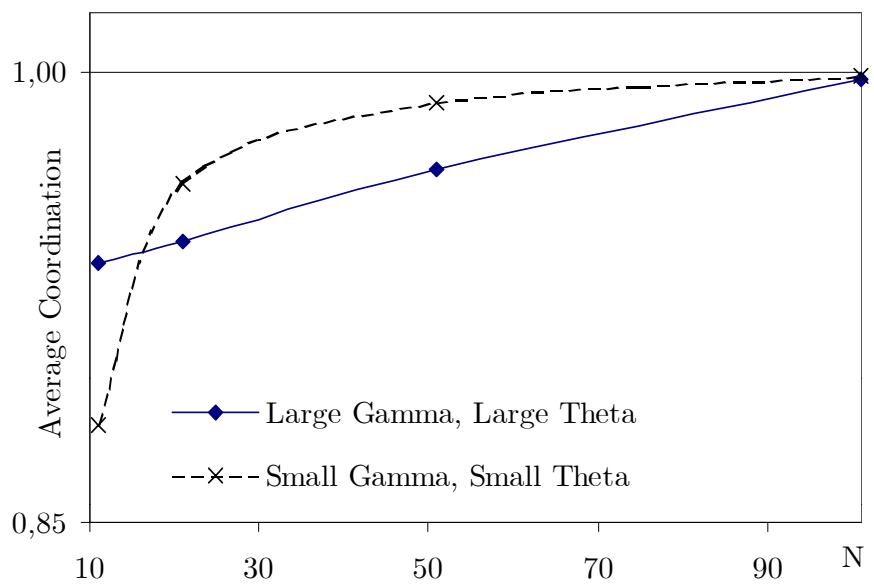

Figure 3(a): Means of MC distributions of average coordination $\bar{c}_{m}$ vs. population size $N$. The Case of Unrestricted Initial Radius Configuration: $r_{i, 0} \in\{1,2, \ldots,\lfloor N / 2\rfloor\}$. Parameter Setup: $\gamma \in\{10 E-07,1.5\}, \theta \in\{0.1,0.9\}, M=1000$. 


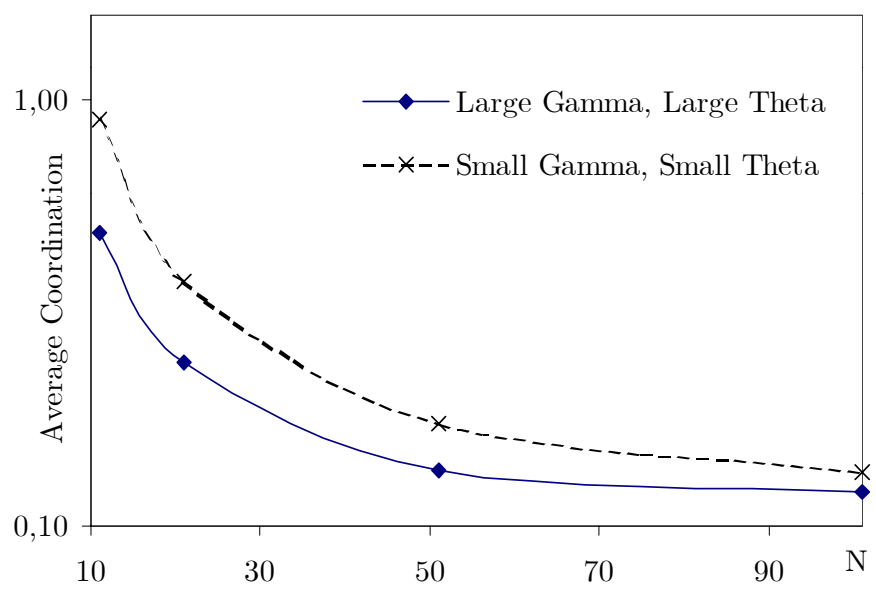

Figure 3(b): Means of MC distributions of average coordination $\bar{c}_{m}$ vs. population size $N$. The Case of Restricted Initial Radius Configuration: $r_{i, 0} \in\{1,2, \ldots, 5\}$. Parameter Setup: $\gamma \in\{10 E-07,1.5\}, \theta \in\{0.1,0.9\}, M=1000$.

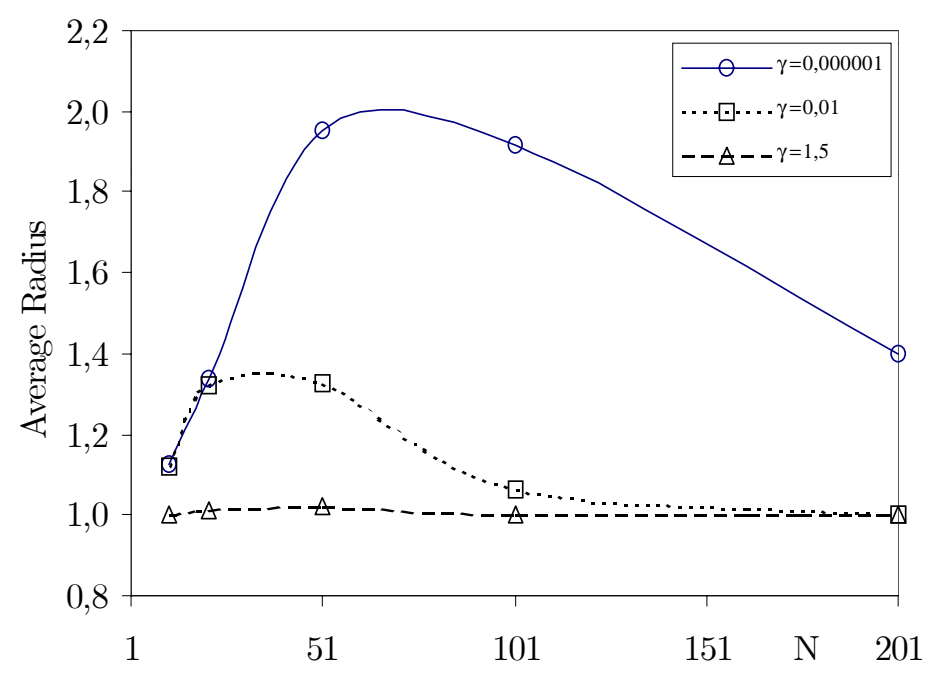

Figure 3(c): Means of MC average radius $\bar{r}_{m}$ vs. population size $N$ for three different values of $\gamma$. Parameter Setup: $\theta=0.9, M=1000$. 


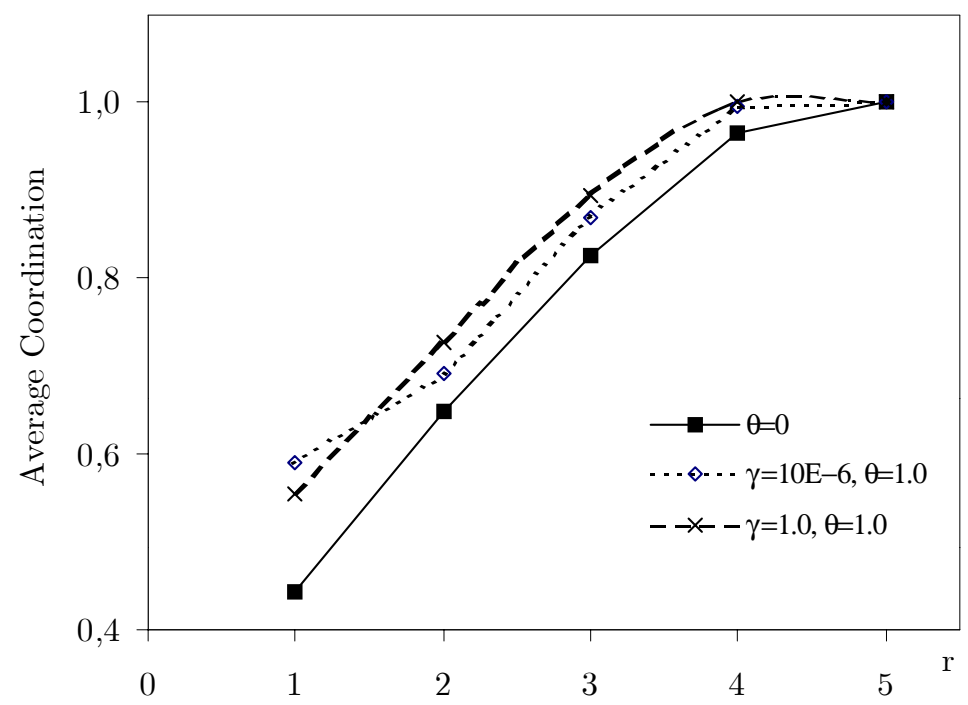

Figure 4(a): Static vs. Endogenous neighborhood structures. The case of homogeneous initial neighborhood radius $r$. An example of the behavior of the means of MC Distributions of Average Coordination $\bar{c}_{m}$ in the case $\theta=0$ vs. $\theta>0$. Solid and dashed lines represent average coordination $\widehat{c}$ as the homogeneous neighborhood radius $r$ increases (Parameter setup: $N=11$, $M=10000)$.

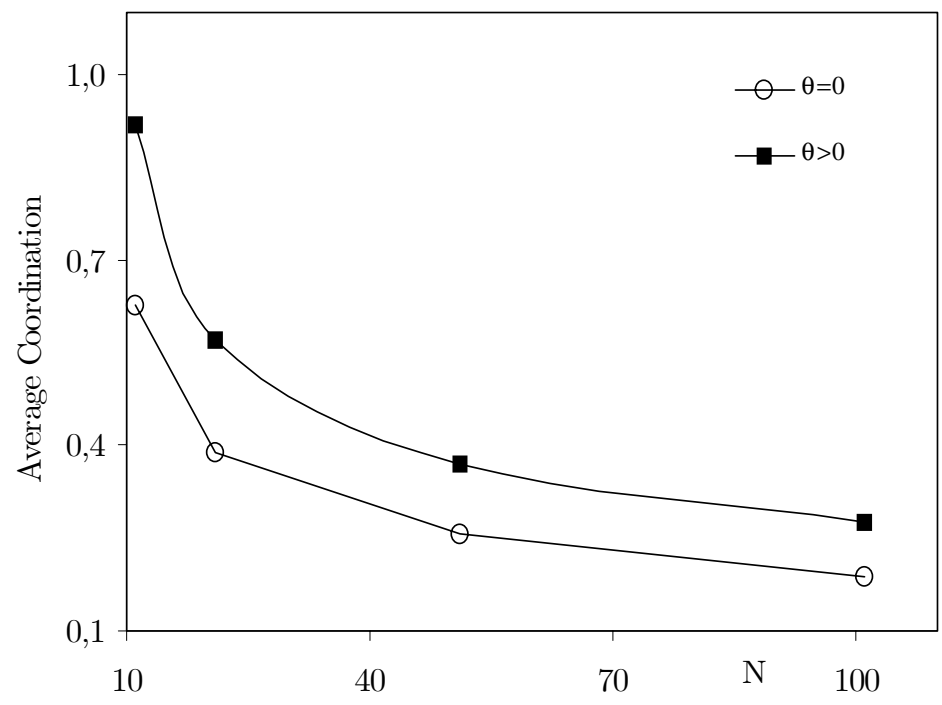

Figure 4(b): Static vs. Endogenous neighborhood structures. The case of homogeneous initial neighborhood radius $r$. An example of the behavior of the means of MC Distributions of Average Coordination $\bar{c}_{m}$ when $\theta=0$ vs. $\theta>0$ as $N$ changes (given $r$ ). Parameter setup: $r_{i, 0}=3$ all $i, M=10000$. Data for $\theta>0$ refer to $\theta=0.1$ and $\gamma=0.001$. 


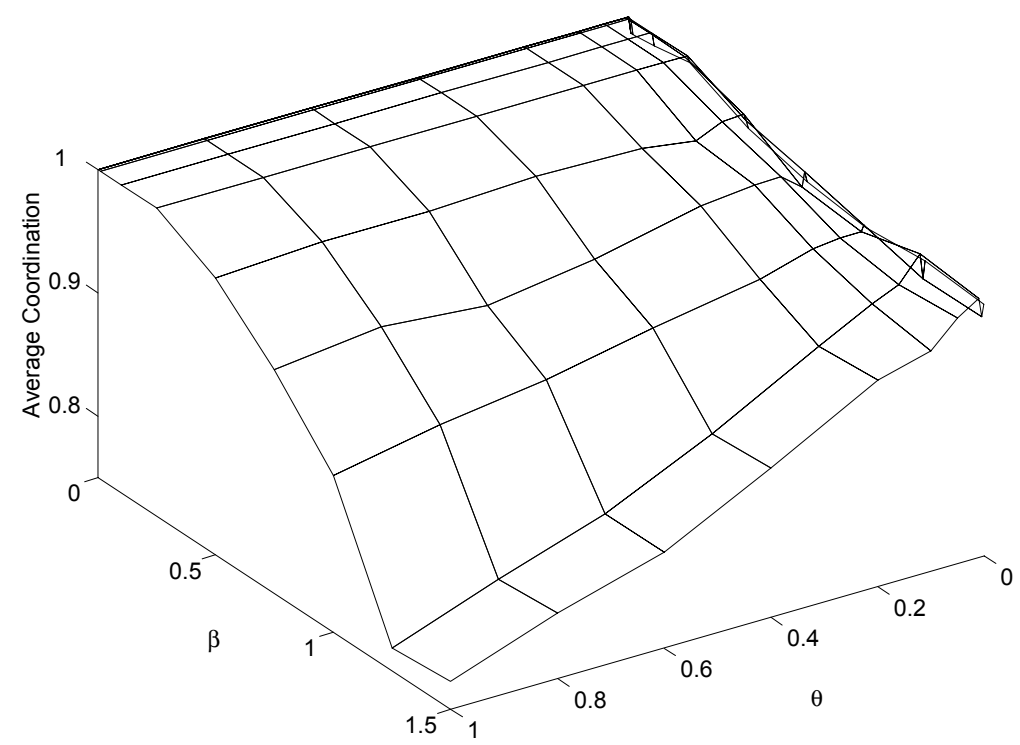

Figure 5(a). The Case of Positive Network Externalities $(\alpha=0)$. Means of MC average coordination distributions $\left\{\bar{c}_{m}, m=1, \ldots, M\right\}$ as a function of the unit neighborhood cost $\beta$ and the frequency of neighborhood updating $\theta$. Population Size $N=21$. MC Sample Size $M=1000$.

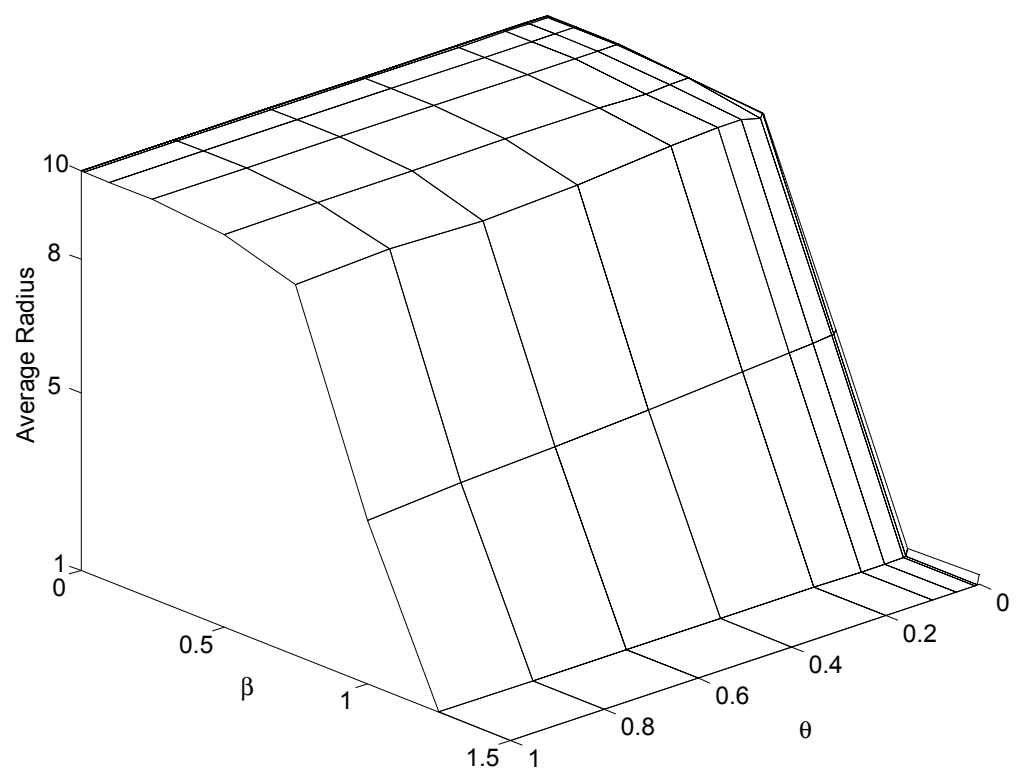

Figure 5(b). The Case of Positive Network Externalities $(\alpha=0)$. Means of MC average radius distributions $\left\{\bar{r}_{m}, m=1, \ldots, M\right\}$ as a function of the unit neighborhood cost $\beta$ and the frequency of neighborhood updating $\theta$. Population Size $N=21$. MC Sample Size $M=1000$. 


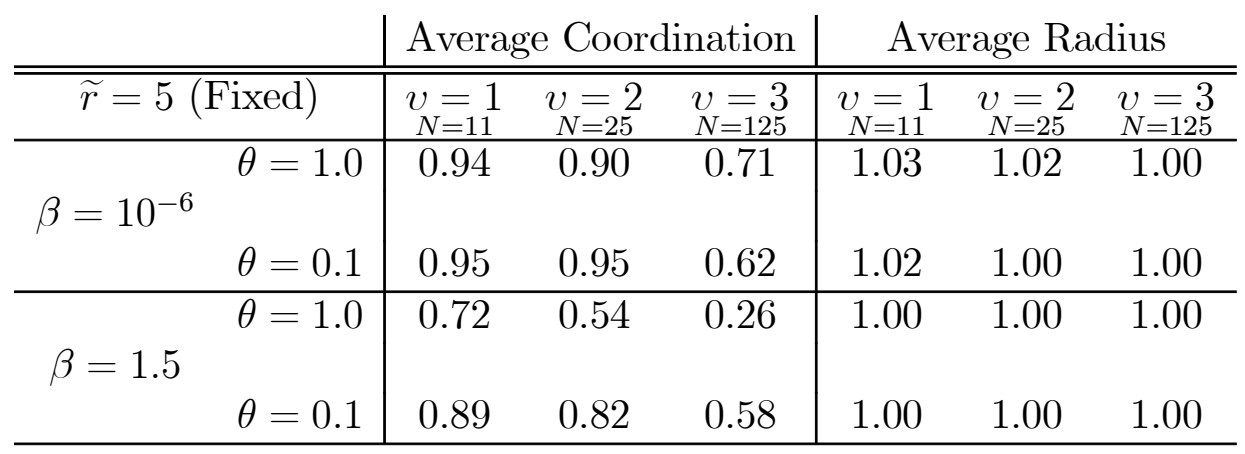

\begin{tabular}{|c|c|c|c|c|c|c|c|}
\hline & & \multicolumn{3}{|c|}{ Average Coordination } & \multicolumn{3}{|c|}{ Average Radius } \\
\hline \multicolumn{2}{|c|}{$N$ Fixed } & $\begin{array}{l}v=1 \\
N=121\end{array}$ & $\begin{array}{l}v=2 \\
N=121\end{array}$ & $\begin{array}{l}v=3 \\
N=125\end{array}$ & $\begin{array}{l}v=1 \\
N=121\end{array}$ & $\begin{array}{l}v=2 \\
N=121\end{array}$ & $\begin{array}{l}v=3 \\
N=125\end{array}$ \\
\hline \multirow{2}{*}{$\beta=10^{-6}$} & $\theta=1.0$ & 0.99 & 0.79 & 0.71 & 1.01 & 1.07 & 1.00 \\
\hline & $\theta=0.1$ & 1.00 & 0.85 & 0.62 & 1.00 & 1.02 & 1.00 \\
\hline \multirow{2}{*}{$\beta=1.5$} & $\theta=1.0$ & 0.97 & 0.41 & 0.26 & 1.00 & 1.00 & 1.00 \\
\hline & $\theta=0.1$ & 0.99 & 0.83 & 0.58 & 1.00 & 1.00 & 1.00 \\
\hline
\end{tabular}

Table 1: MC Means of Average Coordination and Average Radius when agents are located in a $v$-dimensional lattice with periodic boundaries and Von-Neumann Neighborhoods. Top: $\widetilde{r}=N^{1 / v}$ fixed $(=5)$. Bottom: $N$ fixed $(\simeq 125)$. MC Sample Size $M=1000$.

\begin{tabular}{cc|ccc|ccc} 
& \multicolumn{3}{|c|}{ Average Strategy } & \multicolumn{3}{c}{ Average Radius } \\
\hline \hline \multicolumn{2}{c|}{$N=51$} & $c=0.00$ & $c=1.00$ & $c=1.99$ & $c=0.00$ & $c=1.00$ & $c=1.99$ \\
\hline \multirow{2}{*}{$\beta=10^{-6}$} & $\theta=1.0$ & 1.00 & 0.18 & -1.00 & 1.00 & 1.13 & 1.00 \\
& $\theta=0.1$ & 0.84 & -0.03 & -1.00 & 1.00 & 1.01 & 1.00 \\
\hline \multirow{2}{*}{$\beta=1.5$} & $\theta=1.0$ & 1.00 & 0.01 & -1.00 & 1.00 & 1.00 & 1.00 \\
& $\theta=0.1$ & 1.00 & -0.05 & -1.00 & 1.00 & 1.00 & 1.00 \\
\hline
\end{tabular}

Table 2: MC Means of Average Strategy $\left(\bar{s}_{m}\right)$ and Average Radius when the underlying stage-game is a generic coordination game. The parameter $c$ measures the risk-efficiency of $(-1,-1)$. Parameter Setup: $c=0:(+1,+1)$ PE and RD; $c=1:(+1,+1)$ and $(-1,-1)$ risk equivalent; $c=1.99$ : $(-1,-1)$ is RD. MC Sample Size $M=1000$. 